\title{
COHOMOLOGICAL REPRESENTATIONS OF PARAHORIC SUBGROUPS
}

\author{
CHARLOTTE CHAN AND ALEXANDER IVANOV
}

\begin{abstract}
We give a geometric construction of representations of parahoric subgroups $P$ of a reductive group $G$ over a local field which splits over an unramified extension. These representations correspond to characters $\theta$ of unramified maximal tori and, when the torus is elliptic, are expected to give rise to supercuspidal representations of $G$. We calculate the character of these $P$-representations on a special class of regular semisimple elements of $G$. Under a certain regularity condition on $\theta$, we prove that the associated $P$-representations are irreducible. This generalizes a construction of Lusztig from the hyperspecial case to the setting of an arbitrary parahoric.
\end{abstract}

\section{INTRODUCTION}

Let $k$ be a non-archimedean local field with finite residue field. Let $G$ be a reductive group over $k$, and let $T \subseteq G$ be a maximal torus defined over $k$ and split over an unramified extension of $k$. Let $P$ be a parahoric model of $G$, defined over the integers $\mathcal{O}_{k}$. Then $P$ is attached to a point $\mathbf{x}$ in the Bruhat-Tits building $\mathscr{B}_{k}$ of the adjoint group of $G$ over $k$, lying in the apartment of $T$. We denote the schematic closure of $T$ in $P$ again by $T$. We will construct and study a tower of varieties over an algebraic closure of the residue field $\mathbb{F}_{q}$ of $k$ whose cohomology realizes interesting representations of $P\left(\mathcal{O}_{k}\right)$ parametrized by characters of $T\left(\mathcal{O}_{k}\right)$. This construction generalizes classical Deligne-Lusztig theory [DL76] (for reductive groups over finite fields), as well as the work of Lusztig [Lus04 and Stasinski Sta09] (for reductive groups over henselian rings). Further, we give an explicit formula for the character on certain very regular elements, generalizing a special case of the character formula for representations of reductive groups over finite fields [DL76, Theorem 4.2].

More precisely, we work with a Moy-Prasad filtration quotient $\mathbb{G}=\mathbb{G}_{r}(r \geq 1)$ of $P$, regarded as (the perfection of) a smooth affine group scheme of finite type over $\mathbb{F}_{q}$. We normalize this quotients such that $\mathbb{G}_{1}$ is canonically isomorphic to the reductive quotient of the special fiber of $P$. As such, one has a Frobenius $\sigma: \mathbb{G} \rightarrow \mathbb{G}$ and the corresponding Lang map $\mathbb{G} \rightarrow \mathbb{G}, g \mapsto g^{-1} \sigma(g)$. Choose a Borel subgroup of $G$ containing $T$ (defined over some unramified extension of $k$ ) with unipotent radical $U$. In $\mathbb{G}$ we have the subgroups $\mathbb{T}$ and $\mathbb{U}$, corresponding to the closures of $T$ and $U$ in $P$. Consider the subscheme $S_{T, U}=S_{T, U, r} \subset \mathbb{G}$ defined as the preimage of $\mathbb{U}$ under the Lang map. By construction, $S_{T, U}$ has a natural action of $P\left(\mathcal{O}_{k}\right) \times T\left(\mathcal{O}_{k}\right)$ given by left and right multiplication. It factors through an action

Received by the editors October 1, 2019, and, in revised form, November 4, 2020.

2020 Mathematics Subject Classification. Primary 20G25, 14L15.

The first author was partially supported by an NSF Postdoctoral Research Fellowship (DMS1802905) and by the DFG via the Leibniz Prize of Peter Scholze.

The second author was supported by the DFG via the Leibniz Prize of Peter Scholze. 
of $\mathbb{G}\left(\mathbb{F}_{q}\right) \times \mathbb{T}\left(\mathbb{F}_{q}\right)$. For a smooth character $\theta: \mathbb{T}\left(\mathbb{F}_{q}\right) \rightarrow \overline{\mathbb{Q}}_{\ell}^{\times}\left(\ell \neq \operatorname{char} \mathbb{F}_{q}\right)$, we define $R_{T, U}^{\theta}$ to be the $\theta$-isotypic component of the alternating sum of the cohomology groups of $S_{T, U}$ with $\overline{\mathbb{Q}}_{\ell}$-coefficients. This is a virtual $P\left(\mathcal{O}_{k}\right)$-representation.

In Lus04, 1.5] the notion of regularity of a character $\theta: \mathbb{T}_{r}\left(\mathbb{F}_{q}\right) \rightarrow \overline{\mathbb{Q}}_{\ell}^{\times}$is defined for $r \geq 2$. We recall this notion (adapted to our situation) in Section 2.10 below. Roughly speaking, a character is regular if it is "very non-trivial" on $\operatorname{ker}\left(\mathbb{T}_{r}\left(\mathbb{F}_{q}\right) \rightarrow\right.$ $\mathbb{T}_{r-1}\left(\mathbb{F}_{q}\right)$ ). Our first main result is the following generalization of [Lus04, 2.4, 2.5].

Theorem 1.1. Fix an $r \geq 1$ and let $(T, U)$ and $\left(T^{\prime}, U^{\prime}\right)$ be two pairs as above, such that $\mathbf{x}$ lies in the intersection of apartments of $T$ and $T^{\prime}$. Let $\theta: \mathbb{T}\left(\mathbb{F}_{q}\right) \rightarrow \overline{\mathbb{Q}}_{\ell}^{\times}$, $\theta^{\prime}: \mathbb{T}^{\prime}\left(\mathbb{F}_{q}\right) \rightarrow \overline{\mathbb{Q}}_{\ell}^{\times}$be two characters and assume that at least one of $\theta, \theta^{\prime}$ is regular if $r \geq 2$. Then

$$
\left\langle R_{T, U}^{\theta}, R_{T^{\prime}, U^{\prime}}^{\theta^{\prime}}\right\rangle_{\mathbb{G}\left(\mathbb{F}_{q}\right)}=\#\left\{w \in W_{\mathbf{x}}\left(T, T^{\prime}\right)^{\sigma}: \theta \circ \operatorname{Ad}(w)=\theta^{\prime}\right\}
$$

where $W_{\mathbf{x}}\left(T, T^{\prime}\right)=\mathbb{T}_{1}\left(\overline{\mathbb{F}}_{q}\right) \backslash\left\{g \in \mathbb{G}_{1}\left(\overline{\mathbb{F}}_{q}\right):{ }^{g} \mathbb{T}_{1}=\mathbb{T}_{1}^{\prime}\right\}$ is the transporter from $\mathbb{T}_{1}$ to $\mathbb{T}_{1}^{\prime}$ in $\mathbb{G}_{1}$ (a homogeneous space under the Weyl group of $\mathbb{T}_{1}$ in $\mathbb{G}_{1} ;$ cf. Section 2.8 ).

Consequently, if $\theta$ regular, then

(i) $R_{T, U}^{\theta}$ is independent of the choice of $U$.

(ii) If additionally the stabilizer of $\theta$ in $W_{\mathbf{x}}(T, T)^{\sigma}$ is trivial, then $\pm R_{T, U}^{\theta}$ is an irreducible representation of $\mathbb{G}\left(\mathbb{F}_{q}\right)$ (and of $P\left(\mathcal{O}_{k}\right)$ ).

The proof of Theorem 1.1, given in Section 4.2 below, mainly follows the original method of Lusztig [Lus04, who treated the special case when $P$ is reductive over $\mathcal{O}_{k}$. The main idea in Lus04 is as follows: Theorem1.1 reduces to the computation of the $\mathbb{T}\left(\mathbb{F}_{q}\right) \times \mathbb{T}^{\prime}\left(\mathbb{F}_{q}\right)$-equivariant $\ell$-adic Euler characteristic of $\Sigma=\mathbb{G}\left(\mathbb{F}_{q}\right) \backslash S_{T, U} \times$ $S_{T^{\prime}, U^{\prime}}$. Then one partitions $\Sigma$ into locally closed $\mathbb{T}\left(\mathbb{F}_{q}\right) \times \mathbb{T}^{\prime}\left(\mathbb{F}_{q}\right)$-stable varieties in a very subtle way, so that on each such piece, one can construct by hand an action of a connected algebraic group which commutes with the action of $\mathbb{T}\left(\mathbb{F}_{q}\right) \times \mathbb{T}^{\prime}\left(\mathbb{F}_{q}\right)$. The construction of this action is remarkably delicate, and the subtleties here are responsible for the regularity assumption on the character $\theta$.

Let us now describe the technical issue we must tackle in generalizing Lusztig's hyperspecial setting to the general setting. For each $1 \leq s \leq r-1$, we have the unipotent group $\mathbb{G}_{r}^{s}=\operatorname{ker}\left(\mathbb{G}_{r} \rightarrow \mathbb{G}_{s}\right)$. Now, the above-mentioned locally closed decomposition comes from a very particular filtration of $\mathbb{G}_{r}^{1}$ by locally closed subschemes (not subgroups) with subtle properties Lus04, 1.7,1.8]. Its definition uses that the successive quotients $\mathbb{G}_{s}^{s-1}(1<s \leq r)$ are abelian if $P$ is reductive. However, in general, the quotient $\mathbb{G}_{2}^{1}$ need not be abelian (Remark 2.3). This forces us to refine the filtration $\left\{\mathbb{G}_{r}^{s}\right\}_{s}$ of $\mathbb{G}_{r}^{1}$ (Section 4.3) by a filtration of each graded object $\mathbb{G}_{s}^{s-1}$ (for fixed $s$ ) by certain subgroups $H(\varepsilon)(0<\varepsilon \leq 1)$. Roughly speaking, $H(\varepsilon)$ is generated by the " $\mathbb{G}_{s}^{s-1}$-slices" of $T\left(\mathcal{O}_{\breve{k}}\right)$ and of the root subgroups $U_{\alpha}$ of $T$ in $G$ for which the fractional part of the distance of $\mathbf{x}$ to the closest affine root hyperplane with vector part $\alpha$ is $\leq 1-\varepsilon$. The graded pieces of this new filtration are abelian (Lemma 4.4) and moreover satisfy properties (Sections 4.4, 4.5) similar to those in [Lus04, 1.7]. This in turn allows us to define an associated stratification of $\Sigma$ for which we can construct an action of an algebraic group on each piece (Section 4.6). 
Our second result is the computation of traces of unramified very regular elements of $P\left(\mathcal{O}_{k}\right)$ acting on $R_{T, U}^{\theta}$ (Definition 5.1). The proof is based on the Deligne-Lusztig fixed point formula [DL76, Theorem 3.2] and adapts ideas of [DL76, Theorem 4.2].

Theorem 1.2. For any character $\theta: T\left(\mathcal{O}_{k}\right) \rightarrow \overline{\mathbb{Q}}_{\ell}^{\times}$and any unramified very regular element $g \in P\left(\mathcal{O}_{k}\right)$,

$$
\operatorname{Tr}\left(g, R_{T, U}^{\theta}\right)=\sum_{w \in W_{\mathbf{x}}\left(T, Z^{\circ}(g)\right)^{\sigma}}\left(\theta \circ \operatorname{Ad}\left(w^{-1}\right)\right)(g) .
$$

When $G$ is any inner form of $\mathrm{GL}_{n}$ over $k$ and $T$ is an unramified maximal elliptic torus, we prove in CI20 that the Deligne-Lusztig-type set considered by Lusztig in Lus79 is a scheme and its cohomology realizes the compact inductions $\pi_{\theta}$ to $G(k)$ of (an extension of) the $P\left(\mathcal{O}_{k}\right)$-representations $R_{T, U}^{\theta}$. Furthermore, we showcrucially using specializations of both Theorems 1.1 and 1.2 that on the locus of sufficiently generic characters, the correspondence $\theta \mapsto \pi_{\theta}$ is compatible with the composition of the local Langlands and Jacquet-Langlands correspondences.

As such, we expect this work to be closely related to the problem of geometrically constructing representations of $p$-adic groups in general. More specifically, we expect that if $T$ is elliptic and $\theta: T(k) \rightarrow \overline{\mathbb{Q}}_{\ell}^{\times}$is a sufficiently generic character, then the compact induction to $G(k)$ of (an extension of) the $P\left(\mathcal{O}_{k}\right)$-representation $R_{T, U}^{\theta}$ is related to the supercuspidal representations constructed by $\mathrm{Yu} \mathrm{Yu} 01$. Both the irreducibility of and the character formula for $R_{T, U}^{\theta}$ are crucial ingredients to understanding the corresponding $G(k)$-representation within the context of the local Langlands correspondence.

Finally, we make note of the importance of studying these varieties in the present setting of general parahoric subgroups $P$. Already in the setting of inner forms of $\mathrm{GL}_{n}$, it is not enough to study $R_{T, U}^{\theta}$ for reductive $P$; for example, when $G$ is an anisotropic modulo center inner form of $\mathrm{GL}_{n}$, and $T$ unramified elliptic, then the apartment of $T$ in $\mathscr{B}_{k}$ consists of one point, $\mathbf{x}$, and the corresponding parahoric subgroup $P$ is an Iwahori subgroup. This can occur even if $G$ is split: if $G=\mathrm{Sp}_{4}$, then there is a conjugacy class of maximal elliptic tori in $G$, such that the relevant $P$ is non-reductive, with the reductive quotient of the special fiber being isomorphic to $\mathrm{SL}_{2} \times \mathrm{SL}_{2}$.

\section{Preliminaries}

2.1. Notation. We denote by $k$ a non-archimedean local field with residue field $\mathbb{F}_{q}$ of prime characteristic $p$, and by $\breve{k}$ the completion of a maximal unramified extension of $k$. We denote by $\mathcal{O}_{k}, \mathfrak{p}_{k}$ (resp. $\left.\mathcal{O}, \mathfrak{p}\right)$ the integers and the maximal ideal of $k$ (resp. $\breve{k}$ ). The residue field of $\breve{k}$ is an algebraic closure $\overline{\mathbb{F}}_{q}$ of $\mathbb{F}_{q}$. We write $\sigma$ for the Frobenius automorphism of $\breve{k}$, which is the unique $k$-automorphism of $\breve{k}$, lifting the $\mathbb{F}_{q}$-automorphism $x \mapsto x^{q}$ of $\overline{\mathbb{F}}_{q}$. Finally, we denote by $\varpi$ a uniformizer of $k$ (and hence of $\breve{k}$ ) and by ord $=\operatorname{ord}_{\breve{k}}$ the valuation of $\breve{k}$, normalized such that $\operatorname{ord}(\varpi)=1$.

If $k$ has positive characteristic, we let $\mathbb{W}$ denote the ring scheme over $\mathbb{F}_{q}$ where for any $\mathbb{F}_{q}$-algebra $A, \mathbb{W}(A)=A \llbracket \varpi \rrbracket$. If $k$ has mixed characteristic, we let $\mathbb{W}$ denote the $k$-ramified Witt ring scheme over $\mathbb{F}_{q}$ so that $\mathbb{W}\left(\mathbb{F}_{q}\right)=\mathcal{O}_{k}$ and $\mathbb{W}\left(\overline{\mathbb{F}}_{q}\right)=\mathcal{O}$. As the Witt vectors are only well behaved on perfect $\mathbb{F}_{q}$-algebras, algebro-geometric 
considerations when $k$ has mixed characteristic are taken up to perfection. We fix the following convention.

Convention. If $k$ has mixed characteristic, whenever we speak of a scheme over its residue field $\mathbb{F}_{q}$, we mean a perfect scheme, that is a set-valued functor on perfect $\mathbb{F}_{q}$-algebras.

For results on perfect schemes we refer to [Zhu17, BS17]. Note that passing to perfection does not affect the $\ell$-adic étale cohomology; thus for purposes of this paper, we could in principle pass to perfection in all cases. However, in the equal characteristic case working on non-perfect rings does not introduce complications, and we prefer to work in this slightly greater generality.

Fix a prime $\ell \neq p$ and an algebraic closure $\overline{\mathbb{Q}}_{\ell}$ of $\mathbb{Q}_{\ell}$. The field of coefficients of all representations is assumed to be $\overline{\mathbb{Q}}_{\ell}$ and all cohomology groups throughout are compactly supported $\ell$-adic étale cohomology groups.

2.2. Group-theoretic data. We let $G$ be a connected reductive group over $k$, such that the base change $G_{\breve{k}}$ to $\breve{k}$ is split. Let $T$ be a $k$-rational, $\breve{k}$-split maximal torus in $G$. Let $\mathscr{B}_{\breve{k}}$ and $\mathscr{B}_{k}$ denote the Bruhat-Tits building of the adjoint group of $G$ over $\breve{k}$ and over $k$, and let $\mathscr{A}_{T, \breve{k}} \subseteq \mathscr{B}_{\breve{k}}$ denote the apartment of $T$. Note that there is a natural action of $\sigma \in \operatorname{Aut}(\breve{k} / k)$ on $\mathscr{B}_{\breve{k}}$ and on $\mathscr{A}_{T, \breve{k}}$, and that $\mathscr{B}_{k}=\mathscr{B}_{\breve{k}}^{\langle\sigma\rangle}$.

Let $X^{*}(T)$ and $X_{*}(T)$ denote the group of characters and cocharacters of $T$. We denote by $\langle\cdot, \cdot\rangle: X^{*}(T) \times X_{*}(T) \rightarrow \mathbb{Z}$ the natural $\mathbb{Z}$-linear pairing between them. We extend it to the uniquely determined $\mathbb{R}$-linear pairing $\langle\cdot, \cdot\rangle: X^{*}(T)_{\mathbb{R}} \times X_{*}(T)_{\mathbb{R}} \rightarrow \mathbb{R}$, where we write $M_{\mathbb{R}}=M \otimes_{\mathbb{Z}} \mathbb{R}$ for a $\mathbb{Z}$-module $M$.

Denote by $\Phi$ the set of roots of $T$ in $G_{\breve{k}}$ and for a root $\alpha \in \Phi$ let $U_{\alpha} \subseteq G_{\breve{k}}$ denote the corresponding root subgroup. There is an action of $\langle\sigma\rangle$ on $\Phi$. Fix a Chevalley system $u_{\alpha}: \mathbb{G}_{a} \stackrel{\sim}{\rightarrow} U_{\alpha}$ for $G_{\breve{k}}$ (cf. e.g. [BT84, 4.1.3]). To any root $\alpha \in \Phi$ we can attach the valuation $\varphi_{\alpha}: U_{\alpha}(\breve{k}) \rightarrow \mathbb{Z}$ given by $\varphi_{\alpha}\left(u_{\alpha}(y)\right)=\operatorname{ord}(y)$. The set of valuations $\left\{\varphi_{\alpha}\right\}_{\alpha \in \Phi}$ defines a point $\mathbf{x}_{0}$ in the apartment $\mathscr{A}_{T, \breve{k}}$. Moreover $\mathscr{A}_{T, \breve{k}}$ is an affine space under $X_{*}(T)_{\mathbb{R}}$ and the point $\mathbf{x}_{0}+v \in \mathscr{A}_{T, \breve{k}}$ for $v \in X_{*}(T)_{\mathbb{R}}$ corresponds to the valuations $\left\{\widetilde{\varphi}_{\alpha}\right\}_{\alpha \in \Phi}$ of the root datum given by $\widetilde{\varphi}_{\alpha}(u)=\varphi_{\alpha}(u)+\langle\alpha, v\rangle$ (see BT72, 6.2]).

We let $U, U^{-}$be the unipotent radicals of two opposite $\breve{k}$-rational Borel subgroups of $G_{\breve{k}}$ containing $T$.

2.3. Affine roots and filtration on the torus. We have the set $\Phi_{\text {aff }}$ of affine roots of $T$ in $G_{\breve{k}}$. It is the set of affine functions of $\mathscr{A}_{T, \breve{k}}$ defined as

$$
\Phi_{\mathrm{aff}}=\left\{\mathbf{x} \mapsto \alpha\left(\mathbf{x}-\mathbf{x}_{0}\right)+m: \alpha \in \Phi, m \in \mathbb{Z}\right\} .
$$

Denote the affine root $(\alpha, m): \mathbf{x} \mapsto \alpha\left(\mathbf{x}-\mathbf{x}_{0}\right)+m$ and call $\alpha$ its vector part. We have the affine root subgroups $\breve{U}_{\alpha, m} \subseteq U_{\alpha}(\breve{k})$, defined by

$$
\breve{U}_{\alpha, m}=\left\{u \in U_{\alpha}(\breve{k}): u=1 \text { or } \varphi_{\alpha}(u) \geq m\right\}
$$

They define a descending separated filtration of $U_{\alpha}(\breve{k})$. There is a natural action of the Frobenius $\sigma$ on the set of affine roots, determined by $\breve{U}_{\sigma(\alpha, m)}=\sigma\left(\breve{U}_{\alpha, m}\right)$. We make it explicit:

Lemma 2.1. Let $(\alpha, m) \in \Phi_{\text {aff }}$. Then $\sigma(\alpha, m)=\left(\sigma(\alpha), m-\left\langle\alpha, \sigma\left(\mathbf{x}_{0}\right)-\mathbf{x}_{0}\right\rangle\right)$. 
Proof. We have $\sigma(\alpha, m)=\left(\sigma(\alpha), m^{\prime}\right)$ for some $m^{\prime} \in \mathbb{Z}$. The evaluation of the affine-linear form $(\alpha, m)$ on the apartment $\mathcal{A}_{T, \breve{k}}$ is $\sigma$-linear, thus we have for all $\mathbf{x} \in \mathcal{A}_{T, \breve{k}}:$

$$
\begin{aligned}
\sigma(\alpha, m)(\mathbf{x}) & =(\alpha, m)\left(\sigma^{-1}(\mathbf{x})\right)=\left\langle\alpha, \sigma^{-1}(\mathbf{x})-\mathbf{x}_{0}\right\rangle+m \\
& =\left\langle\sigma(\alpha), \mathbf{x}-\mathbf{x}_{0}\right\rangle+m-\left\langle\sigma(\alpha), \sigma\left(\mathbf{x}_{0}\right)-\mathbf{x}_{0}\right\rangle .
\end{aligned}
$$

On the other side, $\left(\sigma(\alpha), m^{\prime}\right)(\mathbf{x})=\left\langle\sigma(\alpha), \mathbf{x}-\mathbf{x}_{0}\right\rangle+m^{\prime}$, hence the lemma.

Let $\widetilde{\mathbb{R}}=\mathbb{R} \cup\{r+: r \in \mathbb{R}\} \cup\{\infty\}$ denote the ordered monoid as in [BT72, 6.4.1]. Let $\breve{T}^{0} \subseteq T(\breve{k})$ be the maximal bounded subgroup. For $r \in \widetilde{\mathbb{R}}_{\geq 0} \backslash\{\infty\}$, we have a descending separated filtration of $\breve{T}^{0}$ given by

$$
\breve{T}^{r}=\left\{t \in \breve{T}^{0}: \operatorname{ord}(\chi(t)-1) \geq r \forall \chi \in X^{*}(T)\right\} .
$$

2.4. Parahoric subgroups, the Moy-Prasad filtration, and integral models. Fix a point $\mathrm{x} \in \mathscr{A}_{T, \breve{k}}$. Following Bruhat and Tits [BT84, 5.2.6], there is a parahoric group scheme $P_{\mathbf{x}}$ over $\mathcal{O}$ attached to $\mathbf{x}$, with generic fiber $G$, and with connected special fiber. The group $\breve{P}_{\mathbf{x}}:=P_{\mathbf{x}}(\mathcal{O})$ is generated by $\breve{T}^{0}$ and $\breve{U}_{\alpha, m}$ for all $(\alpha, m) \in \Phi_{\text {aff }}$ such that $\left\langle\alpha, \mathbf{x}-\mathbf{x}_{\mathbf{0}}\right\rangle \geq-m$ (that is, $\left.(\alpha, m)(\mathbf{x}) \geq 0\right)$. The schematic closure of $T$ in $P_{\mathbf{x}}$ is the connected Néron model of $T$. We denote it again by $T$. We have $T(\mathcal{O})=\breve{T}^{0}$. (As $G_{\breve{k}}$ is split, condition (T) of [Yu15, 8.1] is satisfied. The claim about the closure of $T$ in $P_{\mathbf{x}}$ follows e.g. from Yu15, Corollary 8.6(ii)]. Again, because $G_{\breve{k}}$ is split, it also follows [BT84, 4.6.1] that the connected Néron model of $T$ is equal to the maximal subgroup scheme of finite type of the lft model of $T$. The $\mathcal{O}$-points of the latter are equal to $\breve{T}^{0}$, hence we indeed have $T(\mathcal{O})=\breve{T}^{0}$.)

The Moy-Prasad filtration on $\breve{P}_{\mathbf{x}}$ is given by the series of normal subgroups $\breve{P}_{\mathbf{x}}^{r} \subseteq \breve{P}_{\mathbf{x}}\left(r \in \widetilde{\mathbb{R}}_{\geq 0} \backslash\{\infty\}\right)$, generated by $\breve{T}^{r}$ and $\breve{U}_{\alpha, m}$ for all $(\alpha, m) \in \Phi_{\text {aff }}$ such that $\left\langle\alpha, \mathbf{x}-\mathbf{x}_{0}\right\rangle \geq r-m$. By [Yu15, 8.6 Corollary], there is a unique smooth $\mathcal{O}$ model $P_{\mathbf{x}}^{r}$ of $G$, such that $P_{\mathbf{x}}^{r}(\mathcal{O})=\breve{P}_{\mathbf{x}}^{r}$. Moreover, part (ii) of the same corollary describes the schematic closures of $U_{\alpha}, T$ in $P_{\mathbf{x}}^{r}$, and in particular, we have

$$
\breve{P}_{\mathbf{x}}^{r} \cap U_{\alpha}(\breve{k})=\breve{U}_{\alpha,\left\lceil r-\left\langle\alpha, \mathbf{x}-\mathbf{x}_{0}\right\rangle\right\rceil} \quad \text { and } \quad \breve{P}_{\mathbf{x}}^{r} \cap T(\breve{k})=\breve{T}^{r} .
$$

Note that for $r \in \mathbb{R}_{\geq 0}$, we have $\breve{P}_{\mathbf{x}}^{r+}=\bigcup_{s \in \mathbb{R}, s>r} \breve{P}_{\mathbf{x}}^{s}$. For further properties of the Moy-Prasad filtration we refer to [MP94, §2.6] and for further properties of the smooth models $P_{\mathbf{x}}^{r}$ we refer to $\mathrm{Yu} 15$.

Assume now that $\mathbf{x} \in \mathscr{A}_{T, \breve{k}} \cap \mathscr{B}_{k}$. Then all group schemes $P_{\mathbf{x}}, P_{\mathbf{x}}^{r}$ descend to smooth group schemes over $\mathcal{O}_{k}$, again denoted by $P_{\mathbf{x}}, P_{\mathbf{x}}^{r}$ (cf. [Yu15, §9.1]). In particular, all groups $\breve{P}_{\mathbf{x}}^{r}(r \geq 0)$ are $\sigma$-stable (this can also be deduced from Lemma 2.1. which shows that $\sigma$ maps $\breve{U}_{\alpha,\left\lceil r-\left\langle\alpha, \mathbf{x}-\mathbf{x}_{0}\right\rangle\right\rceil}$ isomorphically onto $\left.\breve{U}_{\sigma(\alpha),\left\lceil r-\left\langle\sigma(\alpha), \mathbf{x}-\mathbf{x}_{0}\right\rangle\right\rceil}\right)$, and $P_{\mathbf{x}}\left(\mathcal{O}_{k}\right)=\breve{P}_{\mathbf{x}}^{\sigma}$ and $P_{\mathbf{x}}^{r}\left(\mathcal{O}_{k}\right)=\left(\breve{P}_{\mathbf{x}}^{r}\right)^{\sigma}$.

2.5. Moy-Prasad quotients. For a scheme $\mathfrak{X}$ over $\mathcal{O}_{k}$ (resp. over $\mathcal{O}$ ), the functor of positive loops $L^{+} \mathfrak{X}$ is the functor on $\mathbb{F}_{q}$-algebras (resp. $\overline{\mathbb{F}}_{q}$-algebras) given by

$$
L^{+} \mathfrak{X}(R)=\mathfrak{X}(\mathbb{W}(R)) .
$$

If $\mathfrak{X}$ is affine and of finite type, then $L^{+} \mathfrak{X}$ is represented by an affine scheme (cf. [PR08, §1.a] if char $k>0$ and [Zhu17, §1] if char $k=0$; for the truncated versions of $L^{+}$, see [Gre61, Gre63]). 
Let $\mathbf{x} \in \mathscr{A}_{T, \breve{k}} \cap \mathscr{B}_{k}$ be as in Section 2.4. We have the infinite-dimensional affine $\mathbb{F}_{q}$-group scheme $L^{+} P_{\mathbf{x}}$, and will now introduce convenient (perfectly) finitely presented quotients of it. Let $r \in \mathbb{Z}_{\geq 1}$. We consider the fpqc quotient sheaf $\mathbb{G}_{r}:=L^{+} P_{\mathbf{x}} / L^{+} P_{\mathbf{x}}^{(r-1)+}$. By CI20, Proposition 4.2(ii)] it is representable by (the perfection of) a smooth affine group scheme over $\mathbb{F}_{q}$ of finite type, which we again denote by $\mathbb{G}_{r}$. From [Yu15, Theorem 8.8], along with the fact that $L^{+} P_{\mathbf{x}}^{(r-1)+}$ is pro-unipotent, it follows by taking Galois cohomology,

$$
\breve{G}_{r}:=\mathbb{G}_{r}\left(\overline{\mathbb{F}}_{q}\right)=\breve{P}_{\mathbf{x}} / \breve{P}_{\mathbf{x}}^{(r-1)+} \quad \text { and } \quad \breve{G}_{r}^{\sigma}=\mathbb{G}_{r}\left(\mathbb{F}_{q}\right)=\left(\breve{P}_{\mathbf{x}} / \breve{P}_{\mathbf{x}}^{(r-1)+}\right)^{\sigma} .
$$

For $r \geq s \geq 1$ we have natural surjections of $\mathbb{F}_{q}$-groups $L^{+} P_{\mathbf{x}} \rightarrow \mathbb{G}_{r} \rightarrow \mathbb{G}_{s}$. We write $\mathbb{G}_{r}^{s}=\operatorname{ker}\left(\mathbb{G}_{r} \rightarrow \mathbb{G}_{s}\right)$ and $\breve{G}_{r}^{s}:=\mathbb{G}_{r}^{s}\left(\overline{\mathbb{F}}_{q}\right)$. Moreover, we also have natural surjections $\mathbb{G}_{2} \rightarrow P_{\mathbf{x}} \otimes_{\mathcal{O}_{k}} \mathbb{F}_{q} \rightarrow\left(P_{\mathbf{x}} \otimes_{\mathcal{O}_{k}} \mathbb{F}_{q}\right)^{\text {red }}=\mathbb{G}_{1}$ identifying $\mathbb{G}_{1}$ with the reductive quotient of the special fiber of $P_{\mathbf{x}}$.

2.6. Subgroups of $\mathbb{G}_{r}$. Let $H \subseteq G_{\breve{k}}$ be a closed subgroup scheme. Let $r \in \mathbb{Z}_{\geq 1}$. We will attach to $H$ the subgroup $\mathbb{H}_{r} \subseteq \mathbb{G}_{r, \overline{\mathbb{F}}_{q}}$ as follows. The schematic closure $H_{\mathbf{x}}$ of $H$ in $P_{\mathbf{x}, \mathcal{O}}$ is flat (by $[\mathrm{BT} 72,1.2 .6]$ as $\mathcal{O}$-flat is equivalent to $\mathcal{O}$-torsion free). It follows that $H_{\mathbf{x}}$ is a closed subgroup scheme of $P_{\mathbf{x}, \mathcal{O}}([\mathrm{BT72}, 1.2 .7])$. Apply $L_{r}^{+}$to the inclusion $H_{\mathbf{x}} \subseteq P_{\mathbf{x}, \mathcal{O}}$ to obtain the subgroup scheme $L_{r}^{+} H_{\mathbf{x}} \subseteq L_{r}^{+} P_{\mathbf{x}, \mathcal{O}}$. The last inclusion is a closed immersion (e.g. by [Gre61, Corollary 2 on p. 639]). We define the closed subgroup scheme $\mathbb{H}_{r} \subseteq \mathbb{G}_{r, \overline{\mathbb{F}}_{q}}$ as the image of $L_{r}^{+} H_{\mathbf{x}}$ under $L_{r}^{+} P_{\mathbf{x}, \mathcal{O}} \rightarrow \mathbb{G}_{r, \overline{\mathbb{F}}_{q}}$. We write $\mathbb{H}_{r}^{s}:=\operatorname{ker}\left(\mathbb{H}_{r} \rightarrow \mathbb{H}_{s}\right)$ and $\mathbb{H}_{r}^{s, *}:=\mathbb{H}_{r}^{s} \backslash \mathbb{H}_{r}^{s+1}$.

Suppose now additionally that $H_{\mathbf{x}}$ is smooth. Then $L_{r}^{+} H_{\mathbf{x}}$ is reduced (one could e.g. use Gre63, Corollary 2 on p. 264]), and hence $\mathbb{H}_{r}$ is too. If $H$ is already defined over the finite subextension of $\breve{k} / k$ of degree $d$, then $H_{\mathbf{x}}$ is defined over the integers of this subextension. This implies that $\mathbb{H}_{r}\left(\overline{\mathbb{F}}_{q}\right)$ is stable under the action of $\sigma^{d}$. Hence $\mathbb{H}_{r}$ is defined over $\mathbb{F}_{q^{d}}$ (here we use that $\mathbb{H}_{r}$ is (the perfection of) a reduced separated scheme of finite type over $\mathbb{F}_{q}$ ).

Using the procedure described above we obtain the closed $\mathbb{F}_{q}$-subgroup $\mathbb{T}_{r} \subseteq$ $\mathbb{G}_{r}$ attached to $T \subseteq G$. Analogously, we have the subgroups $\mathbb{U}_{r}, \mathbb{U}_{r}^{-} \subseteq \mathbb{G}_{r, \overline{\mathbb{F}}_{q}}$ corresponding to $U, U^{-} \subseteq G_{\breve{k}}$ and for any root $\alpha \in \Phi$ the subgroup $\mathbb{U}_{r, \alpha} \subseteq \mathbb{G}_{r, \overline{\mathbb{F}}_{q}}$ corresponding to $U_{\alpha}$. Note that all these are reduced connected closed subgroups of $\mathbb{G}_{r, \overline{\mathbb{F}}_{q}}$. Moreover, $\mathbb{U}_{r, \alpha}$ is defined over $\mathbb{F}_{q^{d}}$ where $d \in \mathbb{Z}_{\geq 1}$ is the smallest positive integer such that $\sigma^{d}(\alpha)=\alpha$ in $\Phi$ (indeed the group $U_{\alpha, \mathbf{x}}$ is smooth by Yu15, 8.3 Theorem (ii)]), and a similar statement holds for $\mathbb{U}_{r}, \mathbb{U}_{r}^{-}$.

For any reduced $\overline{\mathbb{F}}_{q}$-subscheme $\mathbb{X}_{r} \subseteq \mathbb{G}_{r, \overline{\mathbb{F}}_{q}}$, we define $\breve{X}_{r}:=\mathbb{X}_{r}\left(\overline{\mathbb{F}}_{q}\right) \subseteq \mathbb{G}_{r}\left(\overline{\mathbb{F}}_{q}\right)=$ $\breve{G}_{r}$. Thus for example we write $\breve{U}_{\alpha, r}^{a}=\mathbb{U}_{\alpha, r}^{a}\left(\overline{\mathbb{F}}_{q}\right)$ for $\alpha \in \Phi$ and $1 \leq a \leq r-1$. Following Lusztig, we denote by $\mathcal{T}$ the groups $\mathbb{T}_{r}^{r-1}$. For $\alpha \in \Phi$, let $T^{\alpha} \subset T_{\breve{k}} \subset G_{\breve{k}}$ be the unique 1-dimensional torus contained in the subgroup of $G_{\breve{k}}$ generated by $U_{\alpha}$ and $U_{-\alpha}$; let $\mathbb{T}_{r}^{\alpha}$ be the corresponding subgroup scheme of $\mathbb{G}_{r, \overline{\mathbb{F}}_{q}}$ and write $\mathcal{T}^{\alpha}:=\mathbb{T}_{r}^{\alpha, r-1}$.

Lemma 2.2. Let $r \in \mathbb{Z}_{\geq 1}$ and $1 \leq a \leq r-1$.

(i) The group $\breve{G}_{r}$ is generated by $\breve{T}_{r}$ and all $\breve{U}_{\alpha, r}(\alpha \in \Phi)$.

(ii) The group $\breve{G}_{r}^{a+1}$ is generated by $\breve{T}_{r}^{a+1}$ and all $\breve{U}_{\beta, r}^{a+1}(\alpha \in \Phi)$ 
Proof. Both cases follow from [Yu15, Theorem 8.3] applied to the smooth models $P_{\mathbf{x}}$ and $P_{\mathbf{x}}^{a+}$ of $G$ respectively (note that with notations as in loc. cit., the group $G(k)_{\mathbf{x}, f}$ is by definition the one generated by all $\left.U_{a}(k)_{\mathbf{x}, f(a)}\right)$.

Remark 2.3. Let $U^{\prime}$ be the unipotent radical of some other Borel subgroup of $G_{\breve{k}}$ containing $T$. Although $U$ and $U^{\prime}$ are conjugate by an element of $G(\breve{k})$, the groups $\mathbb{U}_{r}\left(\overline{\mathbb{F}}_{q}\right)$ and $\mathbb{U}_{r}^{\prime}\left(\overline{\mathbb{F}}_{q}\right)$ need not be isomorphic. For example, let $G$ be the anisotropic modulo center inner form of $\mathrm{GL}_{3}$ (it splits over $\breve{k}$ and its $k$-points are isomorphic to the units of a division algebra over $k$ ). Let $\mathbf{x}$ be the unique point in $\mathscr{B}_{k}$. Then $\mathbb{G}_{1}=\mathbb{T}_{1}$ is a torus and (after an appropriate choice of $\mathbf{x}_{0}$ ) one has $\mathbb{G}_{2}\left(\overline{\mathbb{F}}_{q}\right)=\left(\begin{array}{ccc}\mathbb{W}_{2}\left(\overline{\mathbb{F}}_{q}\right)^{\times} & \overline{\mathbb{F}}_{q} & \overline{\mathbb{F}}_{q} \\ \varpi \overline{\mathbb{F}}_{q} & \mathbb{W}_{2}\left(\mathbb{\mathbb { F }}_{q}\right)^{\times} & \overline{\mathbb{F}}_{q} \\ \varpi \overline{\mathbb{F}}_{q} & \varpi \overline{\mathbb{F}}_{q} & \mathbb{W}_{2}\left(\overline{\mathbb{F}}_{q}\right)^{\times}\end{array}\right)$, with the multiplication induced by identifying $\overline{\mathbb{F}}_{q}$ with $\mathbb{W}_{1}\left(\overline{\mathbb{F}}_{q}\right), \varpi \overline{\mathbb{F}}_{q}$ with the ideal $\varpi \mathbb{W}_{2}\left(\overline{\mathbb{F}}_{q}\right) \subset \mathbb{W}_{2}\left(\overline{\mathbb{F}}_{q}\right)$, and noting that $\varpi \mathbb{W}_{2}\left(\overline{\mathbb{F}}_{q}\right)$ is naturally a $\mathbb{W}_{1}\left(\overline{\mathbb{F}}_{q}\right)$-module. Now, let $U$ and $U^{\prime}$ be the group of upper- and lower-triangular unipotent matrices in $G$. Then $\mathbb{U}_{2}=\mathbb{U}_{2}^{1}$ is non-abelian, whereas $\mathbb{U}_{2}^{\prime}=\mathbb{U}_{2}^{\prime, 1}$ is abelian.

2.7. The groups $\mathbb{U}_{\alpha, r}$. We now give explicit formulas for $\mathbb{U}_{\alpha, r} \subseteq \mathbb{G}_{r}$.

Definition 2.4. Let $\mathbf{x} \in \mathscr{A}_{T, \breve{k}}$. We call a root $\alpha \in \Phi$

$$
\begin{aligned}
\text { reductive } & \text { if }\left\langle\alpha, \mathbf{x}-\mathbf{x}_{0}\right\rangle \in \mathbb{Z} \\
\text { non-reductive } & \text { otherwise. }
\end{aligned}
$$

For any $\alpha \in \Phi$, we may uniquely write $\left\langle\alpha, \mathbf{x}-\mathbf{x}_{0}\right\rangle=-m_{\alpha}+\varepsilon_{\alpha}$ with $m_{\alpha} \in \mathbb{Z}$ and $0 \leq \varepsilon_{\alpha}<1$. We have $m_{\alpha}=-\left\lfloor\left\langle\alpha, \mathbf{x}-\mathbf{x}_{0}\right\rangle\right\rfloor$.

Note that $\alpha \in \Phi$ is reductive if and only if $\mathbb{U}_{\alpha, 1} \neq 1$.

Lemma 2.5. Let $\mathbf{x} \in \mathscr{A}_{T, \breve{k}}$ and let $r \in \mathbb{Z}_{\geq 1}$. Let $\alpha \in \Phi$. We have

$$
m_{\alpha}+m_{-\alpha}= \begin{cases}0 & \text { if } \alpha \text { is reductive } \\ 1 & \text { otherwise }\end{cases}
$$

Moreover, the natural map $\breve{P}_{\mathbf{x}} \rightarrow \mathbb{G}_{r}\left(\overline{\mathbb{F}}_{q}\right)$ induces

$$
\mathbb{U}_{\alpha, r}\left(\overline{\mathbb{F}}_{q}\right)= \begin{cases}\breve{U}_{\alpha, m_{\alpha}} / \breve{U}_{\alpha, m_{\alpha}+r} & \text { if } \alpha \text { reductive } \\ \breve{U}_{\alpha, m_{\alpha}} / \breve{U}_{\alpha, m_{\alpha}+r-1} & \text { otherwise }\end{cases}
$$

Thus for $a \in \mathbb{Z}, r \geq a \geq 1$, the same map induces

$$
\mathbb{U}_{\alpha, r}^{a}\left(\overline{\mathbb{F}}_{q}\right)= \begin{cases}\breve{U}_{\alpha, m_{\alpha}+a} / \breve{U}_{\alpha, m_{\alpha}+r} & \text { if } \alpha \text { reductive }, \\ \breve{U}_{\alpha, m_{\alpha}+a-1} / \breve{U}_{\alpha, m_{\alpha}+r-1} & \text { otherwise } .\end{cases}
$$

Finally, we have $\mathbb{T}_{r}\left(\overline{\mathbb{F}}_{q}\right)=\breve{T}^{0} / \breve{T}^{r}$ and $\mathbb{T}_{r}^{a}=\breve{T}^{a} / \breve{T}^{r}$.

Proof. Noting that $\lceil-s\rceil=-\lfloor s\rfloor$ for $s \in \mathbb{R}$, the lemma follows immediately from (2.1) and the definitions of $\mathbb{U}_{\alpha, r}, \mathbb{U}_{\alpha, r}^{a}$ and $\mathbb{G}_{r}$.

We have the following elementary lemma will be useful later.

Lemma 2.6. Let $\alpha, \beta \in \Phi$ and assume that $p, q \in \mathbb{Z}_{\geq 1}$, such that $p \alpha+q \beta \in \Phi$. Then $p m_{\alpha}+q m_{\beta}-m_{p \alpha+q \beta}=p \varepsilon_{\alpha}+q \varepsilon_{\beta}-\varepsilon_{p \alpha+q \beta}=\left\lfloor p \varepsilon_{\alpha}+q \varepsilon_{\beta}\right\rfloor$. In particular, $p m_{\alpha}+q m_{\beta}-m_{p \alpha+q \beta} \geq 0$. 
Proof. The first equality is immediate. In particular, $p \varepsilon_{\alpha}+q \varepsilon_{\beta}-\varepsilon_{p \alpha+q \beta}$ is an integer. This, along with the fact that $0 \leq \varepsilon_{p \alpha+q \beta}<1$ by definition, implies the second equality.

2.8. Weyl groups and the Bruhat decomposition. We have the group

$$
W_{\mathbf{x}}(T):=\left(N_{G}(T)(\breve{k}) \cap \breve{P}_{\mathbf{x}}^{0}\right) / \breve{T}^{0}
$$

(cf. HR08, Proposition 8]), and it coincides with the Weyl group $W\left(\mathbb{T}_{1}, \mathbb{G}_{1}\right)$ of the torus $\mathbb{T}_{1}$ in the special fiber $\mathbb{G}_{1}$ of $P_{\mathbf{x}}([$ HR08, Proposition 12]). It follows that both natural maps in the composition

$$
W_{\mathbf{x}}(T) \rightarrow N_{\mathbb{G}_{r}}\left(\mathbb{T}_{r}\right)\left(\overline{\mathbb{F}}_{q}\right) / \mathbb{T}_{r}\left(\overline{\mathbb{F}}_{q}\right) \rightarrow N_{\mathbb{G}_{1}}\left(\mathbb{T}_{1}\right)\left(\overline{\mathbb{F}}_{q}\right) / \mathbb{T}_{1}\left(\overline{\mathbb{F}}_{q}\right)
$$

are isomorphisms. Here $N_{G}(H)$ denotes the scheme-theoretic normalizer of the subgroup $H$ of a group $G$ (note that it might be non-reduced, but we have $N_{G}(H)\left(\overline{\mathbb{F}}_{q}\right)=$ $\left.N_{G}(H)_{\text {red }}\left(\overline{\mathbb{F}}_{q}\right)=\left\{g \in G\left(\overline{\mathbb{F}}_{q}\right): g H g^{-1}=H\right\}\right)$. We also note that $W_{\mathbf{x}}(T)$ coincides with the subgroup of the Weyl group $W=W(T, G)$ of $T$ in $G$ generated by the

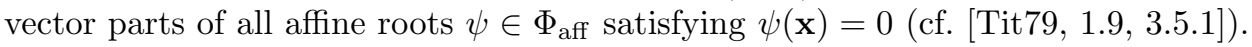
It depends only on the facet of $\mathscr{B}_{\breve{k}}$ in which $\mathbf{x}$ lies, not on $\mathbf{x}$ itself.

We will need a second $k$-rational, $\breve{k}$-split maximal torus $T^{\prime}$ of $G$ whose apartment $\mathscr{A}_{T^{\prime}, \breve{k}}$ in $\mathscr{B}_{\breve{k}}$ passes through the point x. Let $N_{G}\left(T, T^{\prime}\right)=\left\{g \in G: g T g^{-1}=T^{\prime}\right\}$ be the transporter from $T$ to $T^{\prime}$ and analogously, let $N_{\mathbb{G}_{r}}\left(\mathbb{T}_{r}, \mathbb{T}_{r}^{\prime}\right)$ be the transporter from $\mathbb{T}_{r}$ to $\mathbb{T}_{r}^{\prime}$. (Again, these need not be reduced, but we are interested in $\overline{\mathbb{F}}_{q}$-points only.) We then have the principal homogeneous space

$$
W_{\mathbf{x}}\left(T, T^{\prime}\right):=\breve{T}^{0} \backslash\left(N_{G}\left(T, T^{\prime}\right)(\breve{k}) \cap \breve{P}_{\mathbf{x}}^{0}\right)=\mathbb{T}_{r}\left(\overline{\mathbb{F}}_{q}\right) \backslash N_{\mathbb{G}_{r}}\left(\mathbb{T}_{r}, \mathbb{T}_{r}^{\prime}\right)\left(\overline{\mathbb{F}}_{q}\right) .
$$

under $W_{\mathbf{x}}(T)$. Indeed, this follows as $T$ and $T^{\prime}$ are conjugate by an element of $P_{\mathbf{x}}(\mathcal{O})$.

Let $r \geq 1$. For each $w \in W_{\mathbf{x}}\left(T, T^{\prime}\right)$ choose a representative $\dot{w} \in N_{\mathbb{G}_{r}}\left(\mathbb{T}_{r}, \mathbb{T}_{r}^{\prime}\right)\left(\overline{\mathbb{F}}_{q}\right)$, and denote its image in $\mathbb{G}_{1}$ again by $\dot{w}$. We have the Bruhat decomposition $\mathbb{G}_{1}=$ $\bigsqcup_{w \in W_{\mathbf{x}}\left(T, T^{\prime}\right)} \mathbb{G}_{1, w}$ of the reductive quotient, where $\mathbb{G}_{1, w}=\mathbb{U}_{1} \dot{w} \mathbb{T}_{1}^{\prime} \mathbb{U}_{1}^{\prime}$. For $r \geq 1$, define $\mathbb{G}_{r, w}$ to be the pullback of $\mathbb{G}_{1, w}$ along the natural projection $\mathbb{G}_{r} \rightarrow \mathbb{G}_{1}$. Thus $\mathbb{G}_{r}=\bigsqcup_{w \in W_{\mathbf{x}}\left(T, T^{\prime}\right)} \mathbb{G}_{r, w}$. Let $\mathbb{K}_{r}:=\mathbb{U}_{r}^{-} \cap \dot{w} \mathbb{U}_{r}^{\prime-} \dot{w}^{-1}$ and $\mathbb{K}_{r}^{1}:=\mathbb{K}_{r} \cap \mathbb{G}_{r}^{1}$.

Lemma 2.7. For $r \geq 1$, we have $\mathbb{G}_{r, w}=\mathbb{U}_{r} \mathbb{K}_{r}^{1} \dot{w}^{\prime} \mathbb{U}_{r}^{\prime}$.

Proof. We compute

$$
\begin{aligned}
\mathbb{G}_{r, w} & =\mathbb{U}_{r} \dot{w} \mathbb{T}_{r}^{\prime} \mathbb{G}_{r}^{1} \mathbb{U}_{r}^{\prime}=\mathbb{U}_{r} \dot{w} \mathbb{T}_{r}^{\prime}\left(\left(\mathbb{G}_{r}^{1} \cap \mathbb{T}_{r}^{\prime}\right)\left(\mathbb{G}_{r}^{1} \cap \mathbb{U}_{r}^{\prime-}\right)\left(\mathbb{G}_{r}^{1} \cap \mathbb{U}_{r}^{\prime}\right)\right) \mathbb{U}_{r}^{\prime} \\
& =\mathbb{U}_{r} \dot{w} \mathbb{T}_{r}^{\prime}\left(\mathbb{G}_{r}^{1} \cap \mathbb{U}_{r}^{\prime-}\right) \mathbb{U}_{r}^{\prime}=\mathbb{U}_{r}\left(\dot{w}\left(\mathbb{G}_{r}^{1} \cap \mathbb{U}_{r}^{\prime-}\right) \dot{w}^{-1}\right) \dot{w} \mathbb{T}_{r}^{\prime} \mathbb{U}_{r}^{\prime} \\
& =\mathbb{U}_{r}\left(\mathbb{U}_{r}^{-} \cap \dot{w}\left(\mathbb{G}_{r}^{1} \cap \mathbb{U}_{r}^{\prime-}\right) \dot{w}^{-1}\right) \dot{w} \mathbb{T}_{r}^{\prime} \mathbb{U}_{r}^{\prime}=\mathbb{U}_{r} \mathbb{K}_{r}^{1} \dot{w} \mathbb{T}_{r}^{\prime} \mathbb{U}_{r}^{\prime},
\end{aligned}
$$

where the second equality follows from [BT72, 6.4.48].

2.9. Commutation relations. For two subgroups $H_{1}, H_{2}$ of an abstract group $H$, we denote by $\left[H_{1}, H_{2}\right]$ their commutator. For $x, y \in H$, we write $[x, y]:=x^{-1} y^{-1} x y$.

For $\alpha \in \Phi$, let $T^{\alpha} \subseteq T$ denote the image of the coroot corresponding to $\alpha$. It is a one-dimensional subtorus. We also write $\breve{T}^{\alpha, r}=T^{\alpha}(\breve{k}) \cap \breve{T}^{r}$.

Lemma 2.8.

(i) Let $\alpha \in \Phi$ and $r, m \in \widetilde{\mathbb{R}}$. Then $\left[\breve{T}^{r}, \breve{U}_{\alpha, m}\right] \subseteq \breve{U}_{\alpha, m+r}$. 
(ii) If $\alpha, \beta \in \Phi, \alpha \neq-\beta$, and $m_{1}, m_{2} \in \mathbb{Z}$, then $\left[\breve{U}_{\alpha, m_{1}}, \breve{U}_{\beta, m_{2}}\right]$ is contained in the group generated by $\breve{U}_{p \alpha+q \beta, p m_{1}+q m_{2}}$ for all $p, q \in \mathbb{Z}_{\geq 1}$, such that $p \alpha+q \beta \in \Phi$.

(iii) Let $\alpha \in \Phi$ and $m_{1}, m_{2} \in \mathbb{Z}$. Then $\left[\breve{U}_{\alpha, m_{1}}, \breve{U}_{-\alpha, m_{2}}\right] \subseteq \breve{T}^{\alpha, m_{1}+m_{2}}$. For any element $x \in \breve{U}_{-\alpha, m_{2}} \backslash \breve{U}_{-\alpha, m_{2}+1}$, the map $\xi \mapsto[\xi, x]$ induces an isomorphism (of abelian groups)

$$
\lambda_{x}: \breve{U}_{\alpha, m_{1}} / \breve{U}_{\alpha, m_{1}+1} \stackrel{\sim}{\rightarrow} \breve{T}^{\alpha, m_{1}+m_{2}} / \breve{T}^{\alpha, m_{1}+m_{2}+1} .
$$

Proof. (ii) follows from BT72, (6.2.1)]. (i), (iii): By considering a morphism from $\mathrm{SL}_{2}$ to $G_{\breve{k}}$, whose image is generated by $U_{ \pm \alpha}$ (as in [BT72, (6.2.3) b)]), and pulling back the valuation of the root datum along this morphism, it suffices to prove the same statement for $\mathrm{SL}_{2}(\breve{k})$. This is an immediate computation.

For two smooth (connected) closed subgroups $\mathbb{H}_{1}, \mathbb{H}_{2}$ of a connected linear algebraic group $\mathbb{G}$ over a field, we denote by $\left[\mathbb{H}_{1}, \mathbb{H}_{2}\right]$ their commutator "in the sense of group varieties" as in Bor91, §2.3] (it would be more precise to consider the scheme-theoretic commutator, but for our purposes this suffices).

Lemma 2.9. Let $r \geq 2$ and $1 \leq a \leq r-1$. Let $\alpha \in \Phi$.

(a) If $\alpha$ is non-reductive, then $\left[\mathbb{G}_{r}^{a+1}, \mathbb{U}_{\alpha, r}^{r-a}\right]=1$.

(b) If $\alpha$ is reductive, then $\left[\mathbb{G}_{r}^{a}, \mathbb{U}_{r, \alpha}^{r-a}\right]=1$.

Proof. It suffices to prove the claims on $\overline{\mathbb{F}}_{q}$-points.

(a): By Lemma 2.2 it suffices to show to show that $\left[\breve{T}_{r}^{a+1}, \breve{U}_{\alpha, r}^{r-a}\right]=1$ and that $\left[\breve{U}_{\beta, r}^{a+1}, \breve{U}_{\alpha, r}^{r-a}\right]=1(\forall \beta \in \Phi)$ in $\mathbb{G}_{r}$. By Lemma 2.5 $\breve{T}_{r}^{a+1}$ is the image in $\breve{G}_{r}$ of $\breve{T}^{a+1}$, $\breve{U}_{\alpha, r}^{r-a}$ is the image of $\breve{U}_{\alpha, m_{\alpha}+r-a-1}$, and similar claims hold for all $\beta \in \Phi$. But $\left[\breve{T}^{a+1}, \breve{U}_{\alpha, m_{\alpha}+r-a-1}\right] \subseteq \breve{U}_{\alpha, r+m_{\alpha}}$ by Lemma 2.8 (i), and $\breve{U}_{\alpha, r+m_{\alpha}}$ maps to 1 in $\mathbb{G}_{r}$, so $\left[\breve{T}_{r}^{a+1}, \breve{U}_{\alpha, r}^{r-a}\right]=1$ follows. Now assume that $\beta=-\alpha$. Then $-\alpha$ is non-reductive as $\alpha$ is, and by Lemma 2.8(iii), $\left[\breve{U}_{-\alpha, m_{-\alpha}+a}, \breve{U}_{\alpha, m_{\alpha}+r-a-1}\right] \subseteq \breve{T}^{\alpha, r+m_{\alpha}+m_{-\alpha}-1}=\breve{T}^{\alpha, r}$ maps to 1 in $\mathbb{G}_{r}$. This shows $\left[\breve{U}_{-\alpha, r}^{a+1}, \breve{U}_{\alpha, r}^{r-a}\right]=1$. Thus we can assume $\beta \in \Phi$, $\beta \neq-\alpha$. We have two cases.

Case ( $\beta$ is reductive). Then by Lemma 2.5, $\breve{U}_{\beta, r}^{a+1}$ is the image in $\breve{G}_{r}$ of $\breve{U}_{\beta, m_{\beta}+a+1}$ and by Lemma 2.8(ii) we have

$$
\left[\breve{U}_{\beta, m_{\beta}+a+1}, \breve{U}_{\alpha, m_{\alpha}+r-a-1}\right] \subseteq \prod_{\substack{p, q \in \mathbb{Z} \geq 1 \\ p \alpha+q \beta \in \Phi}} \breve{U}_{p \alpha+q \beta, p\left(m_{\alpha}+r-a-1\right)+q\left(m_{\beta}+a+1\right)} .
$$

To ensure that this product maps to 1 in $\breve{G}_{r}$, it suffices to show that for all $p, q \in \mathbb{Z}_{\geq 1}$ with $p \alpha+q \beta \in \Phi$, one has $p\left(m_{\alpha}+r-a-1\right)+q\left(m_{\beta}+a+1\right) \geq m_{p \alpha+q \beta}+r$, or equivalently,

$$
p m_{\alpha}+q m_{\beta}-m_{p \alpha+q \beta}+(p-1)(r-a-1)+(q-1)(a+1) \geq 0 .
$$

But this follows from Lemma 2.6.

Case ( $\beta$ is non-reductive). By Lemma 2.5, $\breve{U}_{\beta, r}^{a+1}$ is the image in $\breve{G}_{r}$ of $\breve{U}_{\beta, m_{\beta}+a}$ and by Lemma 2.8 (ii) we have

$$
\left[\breve{U}_{\beta, m_{\beta}+a}, \breve{U}_{\alpha, m_{\alpha}+r-a-1}\right] \subseteq \prod_{\substack{p, q \in \mathbb{Z}_{\geq 1} \\ p \alpha+q \beta \in \Phi}} \breve{U}_{p \alpha+q \beta, p\left(m_{\alpha}+r-a-1\right)+q\left(m_{\beta}+a\right)} .
$$


To show that the image of this product vanishes in $\mathbb{G}_{r}$, we have to show that each single term does. Assume that $p \alpha+q \beta$ occurs in the product and is non-reductive. Then vanishing of $\breve{U}_{p \alpha+q \beta, p\left(m_{\alpha}+r-a-1\right)+q\left(m_{\beta}+a\right)}$ in $\breve{G}_{r}$ amounts to the inequality

$$
p m_{\alpha}+q m_{\beta}-m_{p \alpha+q \beta}+(p-1)(r-a-1)+(q-1) a \geq 0,
$$

which holds true by Lemma 2.6 Assume finally that $p \alpha+q \beta$ occurs in the product and is reductive. Then vanishing of $\breve{U}_{p \alpha+q \beta, p\left(m_{\alpha}+r-a-1\right)+q\left(m_{\beta}+a\right)}$ in $\breve{G}_{r}$ amounts to the inequality

$$
p m_{\alpha}+q m_{\beta}-m_{p \alpha+q \beta}+(p-1)(r-a-1)+(q-1) a \geq 1,
$$

or equivalently,

$$
\left\lfloor p \varepsilon_{\alpha}+q \varepsilon_{\beta}\right\rfloor+(p-1)(r-a-1)+(q-1) a \geq 1,
$$

i.e. it suffices to show that $p \varepsilon_{\alpha}+q \varepsilon_{\beta} \geq 1$. But as $p \alpha+q \beta$ is reductive,

$(2.2) \mathbb{Z} \ni\left\langle p \alpha+q \beta, \mathbf{x}-\mathbf{x}_{0}\right\rangle=p\left\langle\alpha, \mathbf{x}-\mathbf{x}_{0}\right\rangle+q\left\langle\beta, \mathbf{x}-\mathbf{x}_{0}\right\rangle=-p m_{\alpha}-q m_{\beta}+p \varepsilon_{\alpha}+q \varepsilon_{\beta}$.

As $-p m_{\alpha}-q m_{\beta} \in \mathbb{Z}$, we deduce $p \varepsilon_{\alpha}+q \varepsilon_{\beta} \in \mathbb{Z}$. On the other side $\varepsilon_{\alpha}, \varepsilon_{\beta}>0$ (as $\alpha, \beta$ non-reductive), and hence $p \varepsilon_{\alpha}+q \varepsilon_{\beta}>0$. Thus, $p \varepsilon_{\alpha}+q \varepsilon_{\beta} \geq 1$. This finishes the proof of (a).

(b): We have $\left[\breve{T}^{a}, \breve{U}_{\alpha, m_{\alpha}+r-a}\right] \subseteq \breve{U}_{\alpha, m_{\alpha}+r}$ by Lemma 2.8 (i), and the latter group maps to 1 in $\breve{G}_{r}$. Thus $\left[\breve{T}_{r}^{a}, \breve{U}_{\alpha, r}^{r-a}\right]=1$. Further, Lemma 2.8 (iii) shows

$$
\left[\breve{U}_{-\alpha, m_{-\alpha}+a}, \breve{U}_{\alpha, m_{\alpha}+r-a}\right] \subseteq \breve{T}^{\alpha, m_{\alpha}+m_{-\alpha}+r}=\breve{T}^{\alpha, r},
$$

which maps to 1 in $\breve{G}_{r}$. Thus $\left[\breve{U}_{-\alpha, r}^{a}, \breve{U}_{\alpha, r}^{r-a}\right]=1$. Finally, let $\beta \in \Phi, \beta \neq-\alpha$. Again we have two cases.

Case ( $\beta$ is reductive). By Lemma 2.8 (ii),

$$
\left[\breve{U}_{\beta, m_{\beta}+a}, \breve{U}_{\alpha, m_{\alpha}+r-a}\right] \subseteq \prod_{\substack{p, q \in \mathbb{Z}_{\geq 1} \\ p \alpha+q \beta \in \Phi}} \breve{U}_{p \alpha+q \beta, p\left(m_{\alpha}+r-a\right)+q\left(m_{\beta}+a\right)},
$$

Now, by Lemma 2.6 we have

$$
p\left(m_{\alpha}+r-a\right)+q\left(m_{\beta}+a\right) \geq m_{p \alpha+q \beta}+r .
$$

So, regardless of whether

$$
p \alpha+q \beta
$$

is reductive or not, it follows that $\breve{U}_{p \alpha+q \beta, p\left(m_{\alpha}+r-a\right)+q\left(m_{\beta}+a\right)}$ maps to 1 in $\breve{G}_{r}$, and hence $\left[\breve{U}_{\beta, r}^{a}, \breve{U}_{\alpha, r}^{r-a}\right]=1$.

Case ( $\beta$ is non-reductive). By Lemma 2.8(ii),

$$
\left[\breve{U}_{\beta, m_{\beta}+a-1}, \breve{U}_{\alpha, m_{\alpha}+r-a}\right] \subseteq \prod_{\substack{p, q \in \mathbb{Z}_{\geq 1} \\ p \alpha+q \beta \in \Phi}} \breve{U}_{p \alpha+q \beta, p\left(m_{\alpha}+r-a\right)+q\left(m_{\beta}+a-1\right)},
$$

and the proof can be finished exactly as in the " $\beta$ non-reductive"-case of part (a). 
2.10. Regularity of characters. Recall the notation $\mathcal{T}$ from Section 2.6. Consider the norm map $N_{\sigma}^{\sigma^{m}}: \mathcal{T}\left(\overline{\mathbb{F}}_{q}\right)^{\sigma^{m}} \rightarrow \mathcal{T}\left(\overline{\mathbb{F}}_{q}\right)^{\sigma}=\mathcal{T}\left(\mathbb{F}_{q}\right)$ given by $t \mapsto t \sigma(t) \cdots \sigma^{m-1}(t)$. Let $r \in \mathbb{Z}_{\geq 1}$ be fixed. Following Lusztig [Lus04, 1.5], we say a character $\chi: \mathcal{T}\left(\mathbb{F}_{q}\right) \rightarrow$ $\overline{\mathbb{Q}}_{\ell}^{\times}$is regular if for any $\alpha \in \Phi$ and any $m \geq 1$ such that $\sigma^{m}(\alpha)=\alpha$, the restriction of $\chi \circ N_{\sigma}^{\sigma^{m}}$ to $\mathcal{T}^{\alpha}\left(\overline{\mathbb{F}}_{q}\right)^{\sigma^{m}}$ is non-trivial. A character $\chi$ of $\breve{T}_{r}^{\sigma}$ is called regular if its restriction $\left.\chi\right|_{\mathcal{T}\left(\mathbb{F}_{q}\right)}$ is regular.

Let $\theta: T(k) \rightarrow \overline{\mathbb{Q}}_{\ell}^{\times}$be a character of level $r-1$; that is, $\theta$ is trivial on $\breve{T}^{(r-1)+} \cap$ $T(k)$ but nontrivial on $\breve{T}^{(r-2)+}$. Its restriction to $\breve{T}^{0} \cap T(k)$ can be viewed as a character $\chi$ of $\breve{T}_{r}^{\sigma}=\left(\breve{T}^{0} / \breve{T}^{(r-1)+}\right)^{\sigma}$. We say $\theta$ is regular if $\chi$ is.

Remark 2.10. When $G$ is an inner form of $\operatorname{GL}_{n}(K)$ and $T$ is a maximal nonsplit unramified torus, then $T(k) \cong L^{\times}$, where $L$ is the degree- $n$ unramified extension of $k$. If $\theta: L^{\times} \rightarrow \overline{\mathbb{Q}}_{\ell}^{\times}$is a smooth character trivial on $\left(\breve{T}^{r}\right)^{\sigma}=U_{L}^{r}=1+\varpi^{r} \mathcal{O}_{L}$, then $\theta$ being regular is the same as being primitive in the sense of Boyarchenko-Weinstein BW16, Section 7.1]. This is closely related to $\theta$ being minimal admissible in the sense of Bushnell-Henniart BH05, Section 1.1]. We refer to [CI20, Remark 12.1] for a more precise comparison.

\section{RePresentations of PARAHORIC SUbGroups of $G(k)$}

We use notation from Section 2 . We fix a point $\mathbf{x} \in \mathscr{B}_{k}$, an integer $r \geq 1$, a maximal torus $T$ of $G$ defined over $k$, split over $\breve{k}$, and such that $\mathbf{x} \in \mathscr{A}_{T, \breve{k}}$. Further, we fix the unipotent radicals $U, U^{-}$of opposite Borels containing $T$ in $G_{\breve{k}}$. By construction from Section 2.6, this gives the groups $\mathbb{G}_{r}, \mathbb{T}_{r}, \mathbb{U}_{r}, \mathbb{U}_{r}^{-}$over $\mathbb{F}_{q}$ resp. $\overline{\mathbb{F}}_{q}$.

3.1. The schemes $S_{T, U}$. Let $d$ be the smallest positive integer such that $\sigma^{d}(U)=$ $U$. To this data, we attach the $\mathbb{F}_{q^{d}}$-subscheme of $\mathbb{G}_{r}$,

$$
S_{\mathbf{x}, T, U, r}:=\left\{x \in \mathbb{G}_{r}: x^{-1} \sigma(x) \in \mathbb{U}_{r}\right\} .
$$

To match the notation of Lus04, we write $S_{T, U}$ for $S_{\mathbf{x}, T, U, r}$.

Lemma 3.1. $S_{T, U}$ is separated, and (the perfection of a) smooth scheme of finite type over $\mathbb{F}_{q^{d}}$, which is of dimension $(r-1) \# \Phi^{+}+\# \Phi^{+, \text {red }}$, where $\Phi^{+}$and $\Phi^{+, \text {red }}$ are the roots and reductive roots of $T$ in $U$.

Proof. Indeed, $S_{T, U}$ is the pullback of $\mathbb{U}_{r}$ under the finite étale Lang map $\mathbb{G}_{r} \rightarrow$ $\mathbb{G}_{r}, x \mapsto x^{-1} \sigma(x)$, and $\mathbb{U}_{r}$ is isomorphic to (the perfection of) the affine space of dimension $(r-1) \# \Phi^{+}+\# \Phi^{+, \text {red }}$.

The finite group $\breve{G}_{r}^{\sigma} \times \breve{T}_{r}^{\sigma}$ acts on $S_{T, U}$ by $(g, t): x \mapsto g x t$.

Remark 3.2. $S_{T, U}$ admits also a natural (free) action of $\mathbb{U}_{r} \cap \sigma^{-1}\left(\mathbb{U}_{r}\right)$ by right multiplication. If $r=1$, the quotient of $S_{T, U}$ by this action is $\left(\overline{\mathbb{F}}_{q}\right.$-isomorphic to) a classical Deligne-Lusztig variety for the reductive $\mathbb{F}_{q}$-group $\mathbb{G}_{1}$.

Lemma 3.3. Let $(T, U),\left(T^{\prime}, U^{\prime}\right)$ be two pairs as above (so that, in particular, $\left.\mathbf{x} \in \mathscr{A}_{T, \breve{k}} \cap \mathscr{A}_{T^{\prime}, \breve{k}}\right)$. Then

$$
\breve{G}_{r}^{\sigma} \backslash\left(S_{T, U} \times S_{T^{\prime}, U^{\prime}}\right) \stackrel{\sim}{\rightarrow} \Sigma, \quad\left(g, g^{\prime}\right) \mapsto\left(g^{-1} \sigma(g), g^{\prime-1} \sigma\left(g^{\prime}\right), g^{-1} g^{\prime}\right),
$$

is a $\breve{T}_{r}^{\sigma} \times \breve{T}_{r}^{\prime \sigma}$-equivariant isomorphism, where $\breve{G}_{r}^{\sigma}$ acts diagonally on $S_{T, U} \times S_{T^{\prime}, U^{\prime}}$. 
By functoriality of cohomology, the $\breve{G}_{r}^{\sigma} \times \breve{T}_{r}^{\sigma}$-action on $S_{T, U}$ induces for each $i \in \mathbb{Z}$ a $\breve{G}_{r}^{\sigma} \times \breve{T}_{r}^{\sigma}$-action on $H_{c}^{i}\left(S_{T, U}, \overline{\mathbb{Q}}_{\ell}\right)$. For a character $\theta: \breve{T}_{r}^{\sigma} \rightarrow \overline{\mathbb{Q}}_{\ell}^{\times}$, let $H_{c}^{i}\left(S_{T, U}, \overline{\mathbb{Q}}_{\ell}\right)_{\theta}$ denote the $\theta$-isotypic component. It is stable under the action of $\breve{G}_{r}^{\sigma}$.

Definition 3.4. We define the virtual $\breve{G}_{r}^{\sigma}$-representation with $\overline{\mathbb{Q}}_{\ell}$-coefficients

$$
R_{\mathbf{x}, T, U, r}^{\theta}:=\sum_{i \in \mathbb{Z}}(-1)^{i} H_{c}^{i}\left(S_{T, U}, \overline{\mathbb{Q}}_{\ell}\right)_{\theta} .
$$

By pullback, we can also consider $R_{\mathbf{x}, T, U, r}^{\theta}$ a virtual representation of the parahoric subgroup $\breve{P}_{\mathbf{x}}^{\sigma}$ of $G(k)$. If $\mathbf{x}$ is clear from the context, we write $R_{T, U, r}^{\theta}$ for $R_{\mathbf{x}, T, U, r}^{\theta}$.

Moreover, by Theorem 1.1(i), $R_{T, U, r}^{\theta}$ does not depend on the choice of $U$, if $\theta$ is regular. In this case we denote $R_{T, U, r}^{\theta}$ by $R_{T, r}^{\theta}$. For the dependence on $r$ see Section 3.2 .

Recall the group $N_{\mathbb{G}_{r}}\left(\mathbb{T}_{r}, \mathbb{T}_{r}^{\prime}\right)$ from Section 2.8. Now we generalize [Lus04, 2.2].

Proposition 3.5. Assume that $r \geq 2$. Let $(T, U),\left(T^{\prime}, U^{\prime}\right)$ be two pairs as above. Furthermore, let $\theta: \breve{T}_{r}^{\sigma} \rightarrow \overline{\mathbb{Q}}_{\ell}^{\times}, \theta^{\prime}: \breve{T}_{r}^{\prime \sigma} \rightarrow \overline{\mathbb{Q}}_{\ell}^{\times}$be two characters.

(i) Let $i, i^{\prime} \in \mathbb{Z}$. Assume that an irreducible $\breve{G}_{r}^{\sigma}$-representation appears in the dual space $\left(H_{c}^{i}\left(S_{T, U}, \overline{\mathbb{Q}}_{\ell}\right)_{\theta^{-1}}\right)^{\vee}$ of $H_{c}^{i}\left(S_{T, U}, \overline{\mathbb{Q}}_{\ell}\right)_{\theta}$ and in $H_{c}^{i^{\prime}}\left(S_{T^{\prime}, U^{\prime}}, \overline{\mathbb{Q}}_{\ell}\right)_{\theta^{\prime}}$. Then there exists an integer $n \geq 1$ and a $g \in N_{\mathbb{G}_{r}}\left(\mathbb{T}_{r}^{\prime}, \mathbb{T}_{r}\right)\left(\mathbb{F}_{q^{n}}\right)$ such that the adjoint action of $g$ carries $\left.\theta \circ N_{\sigma}^{\sigma^{n}}\right|_{\mathcal{T}\left(\overline{\mathbb{F}}_{q}\right)^{\sigma^{n}}}$ to $\left.\theta^{\prime} \circ N_{\sigma}^{\sigma^{n}}\right|_{\mathcal{T}^{\prime}\left(\overline{\mathbb{F}}_{q}\right)^{\sigma^{n}}}$.

(ii) Assume that an irreducible $\breve{G}_{r}^{\sigma}$-representation occurs in $R_{T, U, r}^{\theta}$ and $R_{T^{\prime}, U^{\prime}, r}^{\theta^{\prime}}$. Then there exist some $n \geq 1$ and $g \in N_{\mathbb{G}_{r}}\left(\mathbb{T}_{r}^{\prime}, \mathbb{T}_{r}\right)\left(\mathbb{F}_{q^{n}}\right)$ such that the adjoint action of $g$ carries $\left.\theta \circ N_{\sigma}^{\sigma^{n}}\right|_{\mathcal{T}\left(\overline{\mathbb{F}}_{q}\right)^{\sigma^{n}}}$ to $\left.\theta^{\prime} \circ N_{\sigma}^{\sigma^{n}}\right|_{\mathcal{T}^{\prime}\left(\overline{\mathbb{F}}_{q}\right)^{\sigma^{n}}}$.

Proof. The proof (using Lemma 3.3 and Lemma 4.1 below) is literally the same as the proof of [Lus04, Proposition 2.2]. We omit the details.

3.2. Change of level. One could hope that if $\theta$ is a character of $T\left(\mathcal{O}_{k}\right)=\left(\breve{T}^{0}\right)^{\sigma}$ which is trivial on $\left(\breve{T}^{r}\right)^{\sigma}$, then the representations $R_{T, U, r}^{\theta}$ and $R_{T, U, s}^{\theta}$ for all $s \geq r$ coincide. In [CI20, Proposition 7.6], it is shown that this holds when $G$ is an inner form of $\mathrm{GL}_{n}(k)$ and $T$ is an elliptic torus. We will show in subsequent work that for general $G$ which split over $\breve{k}$, this is true when $T$ is elliptic. However this fails for general $T$. In some sense, the more $T$ splits, the bigger is the discrepancy between $R_{T, U, r}^{\theta}$ and $R_{T, U, r+1}^{\theta}$. We will explain the failure in an example.

Assume that $G$ is quasi-split over $k$ and let $T \subseteq G$ be a maximal $k$-rational torus, which contains a $k$-split maximal torus of $G$. Under these assumptions there is a $k$-rational Borel subgroup of $G$ containing $T$. Let $U$ be its unipotent radical. There is a hyperspecial vertex $\mathbf{x}=\mathbf{x}_{0}$ contained in $\mathscr{A}_{T, \breve{k}} \cap \mathscr{B}_{k}$. Let $r \geq 1$, and let $\theta$ be a character of $\left(\breve{T}^{0}\right)^{\sigma}$, which factors through the character (again denoted $\theta$ ) of $\breve{T}_{r}^{\sigma}$. For each $s \geq r$

$$
S_{\mathbf{x}, T, U, s} / \mathbb{U}_{s}=\left(\mathbb{G}_{s} / \mathbb{U}_{s}\right)^{\sigma}=\mathbb{G}_{s}^{\sigma} / \mathbb{U}_{s}^{\sigma}
$$

is a discrete point set. For a surjection of groups $H \rightarrow K$, let $\operatorname{Inf}_{K}^{H}$ denote the inflation functor from virtual $K$-representations to virtual $H$-representations given by pullback. Since $S_{\mathbf{x}, T, U, s}$ and $S_{\mathbf{x}, T, U, s} / \mathbb{U}_{r}$ have the same cohomology groups up 
to an even degree shift, we then have

$$
\begin{aligned}
& R_{\mathbf{x}, T, U, s}^{\theta}=\operatorname{Ind}_{\breve{B}_{s}^{\sigma}}^{\breve{G}^{\sigma}} \operatorname{Inf}_{\breve{B}_{r}^{\sigma}}^{\breve{B}_{s}^{\sigma}} \theta \\
& \operatorname{Inf}_{\breve{G}_{r}^{\sigma}}^{\breve{G}_{s}^{\sigma}} R_{\mathbf{x}, T, U, r}^{\theta}=\operatorname{Inf}_{\breve{G}_{r}^{\sigma}}^{\breve{G}^{\sigma}} \operatorname{Ind}_{\breve{B}_{r}^{\sigma}}^{\breve{G}_{r}^{\sigma}} \theta=\operatorname{Ind}_{\breve{B}_{s}^{\sigma} \breve{G}_{s}^{r, \sigma}}^{\breve{G}^{\sigma}} \operatorname{Inf}_{\breve{B}_{r}^{\sigma}}^{\breve{B}_{s}^{\sigma} \breve{G}_{s}^{r, \sigma}} \theta,
\end{aligned}
$$

where the last formula follows from a general commutativity fact for inflation and induction $\left(\operatorname{Ind}_{H N}^{G} \operatorname{Inf}_{H N / N}^{H N} \chi=\operatorname{Inf}_{G / N}^{G} \operatorname{Ind}_{H N / N}^{G / N} \chi\right.$ for an abstract group $G$, a subgroup $H \subseteq G$, a normal subgroup $N \subseteq G$, and a representation $\chi$ of $H N / N)$. Thus $R_{\mathbf{x}, T, U, s}^{\theta}$ is bigger than $\operatorname{Inf}_{\breve{G}_{r}^{\sigma}}^{\breve{G}_{s}^{\sigma}} R_{\mathbf{x}, T, U, r}^{\theta}$.

\section{THE SCHEME $\Sigma$}

Let the notation be as in the beginning of Section 3. Moreover, let $T^{\prime}$ be another torus such that $\mathrm{x} \in \mathscr{A}_{T, \breve{k}} \cap \mathscr{A}_{T^{\prime}, \breve{k}}$, and let $U^{\prime}, U^{\prime,-}$ be the unipotent radicals of a pair of opposite Borels containing $T^{\prime}$. Let $\mathbb{T}_{r}^{\prime}, \mathbb{U}_{r}^{\prime}, \mathbb{U}_{r}^{\prime,-}$ be the corresponding subgroups of $\mathbb{G}_{r}$.

4.1. Definition of $\Sigma, \Sigma_{w}$. Attached to $(T, U),\left(T^{\prime}, U^{\prime}\right)$, we consider the following locally closed reduced subscheme of $\sigma\left(\mathbb{U}_{r}\right) \times \sigma\left(\mathbb{U}_{r}^{\prime}\right) \times \mathbb{G}_{r}$ whose $\overline{\mathbb{F}}_{q}$-points are given by

$$
\Sigma\left(\overline{\mathbb{F}}_{q}\right):=\left\{\left(x, x^{\prime}, y\right) \in \sigma\left(\breve{U}_{r}\right) \times \sigma\left(\breve{U}_{r}^{\prime}\right) \times \breve{G}_{r}: x \sigma(y)=y x^{\prime}\right\} .
$$

Recalling the Bruhat decomposition discussed in Section 2.8, the scheme $\Sigma$ decomposes into a disjoint union of locally closed subsets $\Sigma=\coprod_{w \in W_{\mathbf{x}}\left(T, T^{\prime}\right)} \Sigma_{w}$, where $\Sigma_{w}$ is the reduced subscheme of $\Sigma$ with $\overline{\mathbb{F}}_{q}$-points

$$
\Sigma_{w}\left(\overline{\mathbb{F}}_{q}\right):=\left\{\left(x, x^{\prime}, y\right) \in \Sigma\left(\overline{\mathbb{F}}_{q}\right): y \in \mathbb{G}_{r, w}\left(\overline{\mathbb{F}}_{q}\right)\right\} .
$$

The group $\breve{T}_{r}^{\sigma} \times \breve{T}_{r}^{\prime \sigma}$ acts on $\Sigma$ and each $\Sigma_{w}$ by

$$
\left(t, t^{\prime}\right):\left(x, x^{\prime}, y\right) \mapsto\left(t x t^{-1}, t^{\prime} x^{\prime} t^{\prime-1}, t y t^{\prime-1}\right) .
$$

The following lemma is completely analogous to [Lus04, Lemma 1.4].

Lemma 4.1. Let $r \geq 2$ and let $\theta: \breve{T}_{r}^{\sigma} \rightarrow \overline{\mathbb{Q}}_{\ell}^{\times}, \theta^{\prime}: \breve{T}_{r}^{\prime \sigma} \rightarrow \overline{\mathbb{Q}}_{\ell}^{\times}$be characters such that $H_{c}^{j}(\Sigma)_{\theta^{-1}, \theta^{\prime}} \neq 0$ for some $j \in \mathbb{Z}$. Then there exist $n \geq 1$ and $g \in N_{\mathbb{G}_{r}}\left(\mathbb{T}_{r}^{\prime}, \mathbb{T}_{r}\right)^{\sigma^{n}}$ such that $\operatorname{Ad}(g)$ carries $\left.\theta\right|_{\mathcal{T}^{\sigma}} \circ N_{\sigma}^{\sigma^{n}}$ to $\left.\theta^{\prime}\right|_{\mathcal{T}^{\prime \sigma}} \circ N_{\sigma}^{\sigma^{n}}$.

Proof. The proof of [Lus04] applies. The only point where one must be careful is the claim that $\mathcal{T}$ and $\mathcal{T}^{\prime}$ centralize $\mathbb{G}_{r}^{1}$ (this is used to extend the action of $\mathcal{T}\left(\mathbb{F}_{q}\right) \times$ $\mathcal{T}^{\prime}\left(\mathbb{F}_{q}\right)$ on a covering of $\Sigma_{w}$ to an action of a connected group). Passing to $\overline{\mathbb{F}}_{q^{-}}$ points, this is the claim that the subgroups $\breve{T}^{(r-2)+} / \breve{T}^{(r-1)+}=\breve{T}^{(r-1)} / \breve{T}^{(r-1)+}$ and $\breve{T}^{\prime(r-2)+} / \breve{T}^{\prime(r-1)+}=\breve{T}^{\prime(r-1)} / \breve{T}^{\prime(r-1)+}$ centralize $\breve{P}_{\mathbf{x}}^{0+} / \breve{P}_{\mathbf{x}}^{(r-1)+}$. By [MP94, 2.6 , end of p.396], we have $\left[P_{\mathbf{x}}^{0+}, P_{\mathbf{x}}^{(r-1)}\right] \subseteq P_{\mathbf{x}}^{(r-1)+}$, which verifies the claim.

4.2. Euler characteristic of $\Sigma$. Fix some $w \in W_{\mathbf{x}}\left(T, T^{\prime}\right)$. Consider the locally closed subscheme $\widehat{\Sigma}_{w}^{\prime}$ of $\sigma\left(\mathbb{U}_{r}\right) \times \sigma\left(\mathbb{U}_{r}^{\prime}\right) \times \mathbb{U}_{r} \times \mathbb{U}_{r}^{\prime} \times\left(\mathbb{K}_{r}^{1} \backslash\{1\}\right) \times \mathbb{T}_{r}^{\prime}$, determined by

$$
\begin{array}{r}
\widehat{\Sigma}_{w}^{\prime}\left(\overline{\mathbb{F}}_{q}\right)=\left\{\left(x, x^{\prime}, u, u^{\prime}, z, \tau^{\prime}\right) \in \sigma\left(\breve{U}_{r}\right) \times \sigma\left(\breve{U}_{r}^{\prime}\right) \times \breve{U}_{r} \times \breve{U}_{r}^{\prime} \times\left(\breve{K}_{r}^{1} \backslash\{1\}\right) \times \breve{T}_{r}^{\prime}:\right. \\
\left.x \sigma\left(u z \dot{w} \tau^{\prime} u^{\prime}\right)=u z \dot{w} \tau^{\prime} u^{\prime} x^{\prime}\right\},
\end{array}
$$


and define an action of $\breve{T}_{r}^{\sigma} \times \breve{T}_{r}^{\prime \sigma}$ on it by

$$
\left(t, t^{\prime}\right):\left(x, x^{\prime}, u, u^{\prime}, z, \tau^{\prime}\right) \mapsto\left(t x t^{-1}, t^{\prime} x^{\prime} t^{\prime-1}, t u t^{-1}, t^{\prime} u^{\prime} t^{\prime-1}, t z t^{-1}, \dot{w}^{-1} t \dot{w} \tau^{\prime} t^{\prime-1}\right) .
$$

Generalizing [Lus04, 1.9(c)], we will show the following proposition, which is the main technical result of Section 4 .

Proposition 4.2. Let $\theta$ and $\theta^{\prime}$ be characters of $\breve{T}_{r}^{\sigma}$ and $\breve{T}_{r}^{\prime \sigma}$ respectively, and assume that $\theta$ or $\theta^{\prime}$ is regular. For each $w \in W_{\mathbf{x}}\left(T, T^{\prime}\right)$, we have

$$
\sum_{i \in \mathbb{Z}}(-1)^{i} \operatorname{dim} H_{c}^{i}\left(\widehat{\Sigma}_{w}^{\prime}, \overline{\mathbb{Q}}_{\ell}\right)_{\theta^{-1}, \theta^{\prime}}=0 .
$$

We prove Proposition 4.2 in Section 4.6] after the necessary preparations. As a corollary to Proposition 4.2, we deduce the following analogue of [Lus04, Lemma $1.9]$ and use it to prove Theorem 1.1 .

Corollary 4.3. With assumptions as in Proposition 4.2, we have

$$
\sum_{i \in \mathbb{Z}} \operatorname{dim} H_{c}^{i}\left(\Sigma, \overline{\mathbb{Q}}_{\ell}\right)_{\theta^{-1}, \theta^{\prime}}=\#\left\{w \in W_{\mathbf{x}}\left(T, T^{\prime}\right)^{\sigma}: \theta \circ \operatorname{Ad}(\dot{w})=\theta^{\prime}\right\} .
$$

Proof. The proof goes along the lines of the proof of [Lus04, 1.9] (all arguments except for the proof of Proposition 4.2 are literally the same).

Proof of Theorem 1.1. The case $r=1$ is equivalent to the classical Deligne-Lusztig orthogonality relations [DL76, Theorem 6.8] for the reductive group $\mathbb{G}_{1}$ over $\mathbb{F}_{q}$. Suppose now that $r \geq 2$. For the first statement of Theorem 1.1 observe that a standard computation using Lemma 3.3 and the Künneth formula shows that $\left\langle R_{T, U}^{\theta}, R_{T^{\prime}, U^{\prime}}^{\theta^{\prime}}\right\rangle=\sum_{i \in \mathbb{Z}} \operatorname{dim} H_{c}^{i}\left(\Sigma, \overline{\mathbb{Q}}_{\ell}\right)_{\theta^{-1}, \theta^{\prime}}$. Now apply Corollary 4.3. Now statements (i) and (ii) follow from the already proven part as in [Lus04, 2.4].

4.3. Filtration of $\mathbb{G}_{a+1}^{a}$. The main difference between the present article and Lus04 is that if $P_{\mathbf{x}}$ is not reductive (i.e. if $\mathbf{x}$ is not a hyperspecial point), then $\mathbb{G}_{2}^{1}$ may not be abelian. This is significant because Lusztig's construction of a stratification of $\widehat{\Sigma}_{w}$ and a corresponding action of a connected algebraic group [Lus04, 1.7,1.8] depend on the abelianness of $\mathbb{G}_{a+1}^{a}$. To deal with this problem, we need a refinement of the filtration of $\mathbb{G}_{r}^{1}$ by its subgroups $\mathbb{G}_{r}^{a}$ for $1 \leq a \leq r-1$. For $a \geq 1$, we define a filtration of $\mathbb{G}_{a+1}^{a}$ as follows: let

$$
H(1):=\text { subgroup of } \mathbb{G}_{a+1}^{a} \text { generated } \mathbb{T}_{a+1}^{a} \text { and } \mathbb{U}_{\alpha, a+1}^{a} \text { for all reductive } \alpha \in \Phi,
$$

and for all $0 \leq \varepsilon<1$, let

$$
\begin{array}{r}
H(\varepsilon):=\text { subgroup of } \mathbb{G}_{a+1}^{a} \text { generated by } H(1) \text { and all } \mathbb{U}_{\alpha, a+1}^{a} \text { for } \alpha \in \Phi, \\
\text { satisfying } \varepsilon_{\alpha} \geq \varepsilon .
\end{array}
$$

Note that $\mathbb{T}_{a+1}^{a} \subseteq H(1) \subseteq H\left(\varepsilon^{\prime}\right) \subseteq H(\varepsilon) \subseteq \mathbb{G}_{a}^{a+1}$ for all $1>\varepsilon^{\prime} \geq \varepsilon>0$. Moreover, there are only finitely many values of $\varepsilon$ ("jumps") satisfying $H(\varepsilon) \supsetneq \bigcup_{\varepsilon^{\prime}>\varepsilon} H\left(\varepsilon^{\prime}\right)$. We denote these jumps by $1=: \varepsilon_{s+1}>\varepsilon_{s}>\cdots>\varepsilon_{1}>0$ for some $s \geq 0$ (thus 1 is a jump by definition). The jumps are independent of $a$. We have $H\left(\varepsilon_{1}\right)=\mathbb{G}_{a+1}^{a}$. For $a \leq r-1$, let $p: \mathbb{G}_{r}^{a} \rightarrow \mathbb{G}_{a+1}^{a}$ be the natural projection, and for $s+1 \geq i \geq 1$, put

$$
\mathbb{G}_{r}^{a, i}:=p^{-1}\left(H\left(\varepsilon_{i}\right)\right) .
$$


For convenience, we put $\mathbb{G}_{r}^{a, s+2}:=\mathbb{G}_{r}^{a+1}$. This defines a refinement $\left\{\mathbb{G}_{r}^{a, i}\right\}_{\substack{r-1 \geq a \geq 1 \\ s+2 \geq i \geq 1}}$ of the filtration $\left\{\mathbb{G}_{r}^{a}\right\}_{r-1 \geq a \geq 1}$ of $\mathbb{G}_{r}^{1}$, decreasing with respect to the lexicographical ordering on pairs $(a, i)$. For $s+1 \geq i \geq 1$, let $\Phi_{i}$ be the set of roots "appearing" in $H\left(\varepsilon_{i}\right) / H\left(\varepsilon_{i+1}\right)$ :

$$
\Phi_{i}:= \begin{cases}\left\{\alpha \in \Phi: \varepsilon_{\alpha}=0\right\} & \text { if } i=s+1 \\ \left\{\alpha \in \Phi: \varepsilon_{\alpha}=\varepsilon_{i}\right\} & \text { if } s \geq i \geq 1 .\end{cases}
$$

Lemma 4.4. Let $r \geq 2$ and $r-1 \geq a \geq 1$.

(i) Let $a \geq 2$. Then $\mathbb{G}_{r}^{a} / \mathbb{G}_{r}^{a+1}=\mathbb{G}_{a+1}^{a}$ is abelian, and in particular, for $s+1 \geq$ $i \geq 1, \mathbb{G}_{r}^{a, i} / \mathbb{G}_{r}^{a, i+1}$ is abelian.

(ii) Let $a=1$ and $s+1 \geq i \geq 1$. Then $\mathbb{G}_{r}^{1, i}$ is normal in $\mathbb{G}_{r}^{1}$ and the quotient $\mathbb{G}_{r}^{1, i} / \mathbb{G}_{r}^{1, i+1}$ is abelian.

Proof. It suffices to prove the assertions on $\overline{\mathbb{F}}_{q}$-points. To show (i), notice that if $a \geq 2$, then $\left[\breve{P}_{\mathbf{x}}^{(a-1)+}, \breve{P}_{\mathbf{x}}^{(a-1)+}\right] \subseteq \breve{P}_{\mathbf{x}}^{2(a-1)+} \subseteq \breve{P}_{\mathbf{x}}^{a+}$, so it follows that $\breve{G}_{a+1}^{a}=$ $\breve{P}_{\mathbf{x}}^{(a-1)+} / \breve{P}_{\mathbf{x}}^{a+}$ is abelian. To establish (ii), it is enough to show that (with $a=1$ ) for any $s+1 \geq i \geq 1, H\left(\varepsilon_{i}\right)$ is normal in $\mathbb{G}_{2}^{1}$ and that $H\left(\varepsilon_{i}\right) / H\left(\varepsilon_{i+1}\right)$ is abelian. We spend the rest of the proof establishing these two claims. Recall that for $s+1 \geq$ $i \geq 1, H\left(\varepsilon_{i}\right)$ is generated by $\mathbb{T}_{2}^{1}$ and all $\mathbb{U}_{\alpha, 2}^{1}$ with $\alpha \in \bigsqcup_{j=i}^{s+1} \Phi_{j}$.

We start with $i=s+1$, i.e. the case $H\left(\varepsilon_{s+1}\right)=H(1)$. By Lemma $2.8 .\left[\breve{T}_{2}^{1}, \breve{U}_{\alpha, 2}^{1}\right]=$ 1. Let $\alpha \in \Phi_{s+1}$ (thus $\alpha$ is reductive) and let $\beta \in \Phi$ be any non-reductive root. Then $\left[\breve{U}_{\alpha, 2}^{1}, \breve{U}_{\beta, 2}^{1}\right]$ is the image in $\breve{G}_{2}^{1}$ of

$$
\left[\breve{U}_{\alpha, m_{\alpha}+1}, \breve{U}_{\beta, m_{\beta}}\right] \subseteq \prod_{\substack{p, q \in \mathbb{Z}_{\geq 1} \\ p \alpha+q \beta \in \Phi}} \breve{U}_{p \alpha+q \beta, p\left(m_{\alpha}+1\right)+q m_{\beta}} .
$$

Using Lemma 2.6 along with $p \geq 1$, we see that $p\left(m_{\alpha}+1\right)+q m_{\beta} \geq m_{p \alpha+q \beta}+1$. Thus the contribution of $p \alpha+q \beta$ to the commutator lies in $\breve{U}_{p \alpha+q \beta, m_{p \alpha+q \beta}+1}$. From this we deduce $\left[\mathbb{U}_{\alpha, 2}^{1}, \mathbb{U}_{\beta, 2}^{1}\right] \subseteq H(1)$. Thus if $x \in \mathbb{U}_{\beta, 2}^{1}$ for any $\beta \in \Phi$, and $y \in \mathbb{U}_{\alpha, 2}^{1}$, then $x y x^{-1}=\left[x^{-1}, y^{-1}\right] y \in H(1)$, which shows that $H(1)$ is normal in $\mathbb{G}_{2}^{1}$. A computation analogous to (4.3) for $\alpha, \beta \in \Phi^{+}$both reductive, shows immediately that $\left[\mathbb{U}_{\alpha, 2}^{1}, \mathbb{U}_{\beta, 2}^{1}\right]=1$ and $\left[\mathbb{T}_{2}^{1}, \mathbb{U}_{\alpha, 2}^{1}\right]=1$, so $H(1)$ is abelian.

Next, pick some $s \geq i \geq 1$. We show that $H\left(\varepsilon_{i}\right)$ is normal in $\mathbb{G}_{2}^{1}$. Since we have already established that $H\left(\varepsilon_{s+1}\right)$ is normal in $\mathbb{G}_{2}^{1}$, it suffices to check as above that for all (non-reductive) $\alpha \in \Phi$ with $\varepsilon_{\alpha} \geq \varepsilon_{i}$ and all non-reductive $\beta \in \Phi$, we have $\left[\mathbb{U}_{\alpha, 2}^{1}, \mathbb{U}_{\beta, 2}^{1}\right] \subseteq H\left(\varepsilon_{i}\right)$. Now, $\left[\breve{U}_{\alpha, 2}^{1}, \breve{U}_{\beta, 2}^{1}\right]$ is the image in $\breve{G}_{2}^{1}$ of

$$
\left[\breve{U}_{\alpha, m_{\alpha}}, \breve{U}_{\beta, m_{\beta}}\right] \subseteq \prod_{\substack{p, q \in \mathbb{Z}_{\geq 1} \\ p \alpha+q \beta \in \Phi}} \breve{U}_{p \alpha+q \beta, p m_{\alpha}+q m_{\beta}} .
$$

Now, if $\varepsilon_{p \alpha+q \beta} \geq \varepsilon_{i}$, then the contribution of $p \alpha+q \beta$ to the commutator is contained in $\mathbb{U}_{p \alpha+q \beta, 2}^{1} \subseteq H\left(\varepsilon_{i}\right)$. If $p \alpha+q \beta$ is reductive, the same computation as in (2.2) shows that $\breve{U}_{p \alpha+q \beta, p m_{\alpha}+q m_{\beta}} \subseteq \breve{U}_{p \alpha+q \beta, m_{p \alpha+q \beta}} \rightarrow \breve{U}_{p \alpha+q \beta, 2}^{1}$. It remains to handle the case that $p \alpha+q \beta$ is non-reductive with $\varepsilon_{p \alpha+q \beta}<\varepsilon_{i}$. If $p \varepsilon_{\alpha}+q \varepsilon_{\beta}<1$, then by Lemma 2.6. $p \varepsilon_{\alpha}+q \varepsilon_{\beta}-\varepsilon_{p \alpha+q \beta}=\left\lfloor p \varepsilon_{\alpha}+q \varepsilon_{\beta}\right\rfloor=0$, i.e. $\varepsilon_{i}>\varepsilon_{p \alpha+q \beta}=p \varepsilon_{\alpha}+q \varepsilon_{\beta} \geq p \varepsilon_{i}$, which is a contradiction. Thus we must have $p \varepsilon_{\alpha}+q \varepsilon_{\beta} \geq 1$, hence $p m_{\alpha}+q m_{\beta}-m_{p \alpha+q \beta}=$ $\left\lfloor p \varepsilon_{\alpha}+q \varepsilon_{\beta}\right\rfloor \geq 1$. Thus $\breve{U}_{p \alpha+q \beta, p m_{\alpha}+q m_{\beta}} \subseteq \breve{U}_{p \alpha+q \beta, m_{p \alpha+q \beta}+1}$, whose image in $\breve{G}_{2}^{1}$ 
vanishes. We may finally conclude that $\left[\mathbb{U}_{\alpha, 2}^{1}, \mathbb{U}_{\beta, 2}^{1}\right] \subseteq H\left(\varepsilon_{i}\right)$, which finishes the proof of normality of $H\left(\varepsilon_{i}\right)$ in $\mathbb{G}_{2}^{1}$.

For $\alpha$ and $\beta$ non-reductive with $\varepsilon_{\alpha}=\varepsilon_{\beta}=\varepsilon_{i}$, a similar computation shows that $\left[\mathbb{U}_{\alpha, 2}^{1}, \mathbb{U}_{\beta, 2}^{1}\right] \subseteq H\left(\varepsilon_{i+1}\right)$. Thus $H\left(\varepsilon_{i}\right) / H\left(\varepsilon_{i+1}\right)$ is abelian.

4.4. Pairings induced by the commutator. Let $N, N^{-}$be the unipotent radicals of any two opposite Borel subgroups of $G$ which contain $T$ and are defined over $\breve{k}$. (We will specify $N$ to suit our needs in Section 4.6.) For $r-1 \geq a \geq 1$, let $\mathbb{N}_{r}, \mathbb{N}_{r}^{-}$and $\mathbb{N}_{r}^{a}, \mathbb{N}_{r}^{-}, a$ be the corresponding subgroups of $\mathbb{G}_{r}$ and $\mathbb{G}_{r}^{a}$. Let $\Phi^{+}=\left\{\alpha \in \Phi: \mathbb{U}_{\alpha, r} \subseteq \mathbb{N}_{r}\right\}$ and $\Phi^{-}=\Phi \backslash \Phi^{+}=\left\{\alpha \in \Phi: \mathbb{U}_{\alpha, r} \subseteq \mathbb{N}_{r}^{-}\right\}$. For $s+1 \geq i \geq 1$, set $\Phi_{i}^{+}=\Phi_{i} \cap \Phi^{+}$and $\Phi_{i}^{-}=\Phi_{i} \cap \Phi^{-}$, and let $\mathbb{N}_{r}^{1, i}=\mathbb{G}_{r}^{1, i} \cap \mathbb{N}_{r}$. We study some pairings induced by the commutator map. Note that the targets of the maps in Lemma 4.5 are abelian by Lemma 4.4 .

Lemma 4.5. Let $r \geq 2$ and $r-1 \geq a \geq 1$. Let $\alpha \in \Phi$ be a non-reductive root.

(i) Let $a \geq 2$. The commutator map induces a bilinear pairing of abelian groups,

$$
\mathbb{U}_{\alpha, r}^{r-a} / \mathbb{U}_{\alpha, r}^{r-a+1} \times \mathbb{N}_{r}^{a} / \mathbb{N}_{r}^{a+1} \rightarrow \mathbb{G}_{r}^{r-1}, \quad(\bar{\xi}, \bar{x}) \mapsto[\bar{\xi}, \bar{x}] .
$$

(ii) Let $a=1$ and $s+1 \geq i \geq 1$. Assume that $\varepsilon_{-\alpha}=\varepsilon_{i}$ (thus $\varepsilon_{\alpha}=1-\varepsilon_{i}$ ). We have $\left[\mathbb{U}_{\alpha, r}^{r-1}, \mathbb{N}_{r}^{1, i}\right] \subseteq \mathbb{G}_{r}^{r-1, s+1}$ and $\left[\mathbb{U}_{\alpha, r}^{r-1}, \mathbb{N}_{r}^{1, i+1}\right]=1$. The commutator map induces a bilinear pairing of abelian groups,

$$
\mathbb{U}_{\alpha, r}^{r-1} \times \mathbb{N}_{r}^{1, i} / \mathbb{N}_{r}^{1, i+1} \rightarrow \mathbb{G}_{r}^{r-1, \mathrm{~s}+1}, \quad(\xi, \bar{x}) \mapsto[\bar{\xi}, \bar{x}]
$$

Proof.

(i): By Lemma 2.9 applied three times, the commutator map $\mathbb{U}_{\alpha, r}^{r-a} \times \mathbb{N}_{r}^{a} \rightarrow \mathbb{G}_{r}$ induces the claimed pairing. It is linear in $\bar{x}$ : if $x_{1}, x_{2} \in \breve{N}_{r}^{a}$, then

$\left[\xi, x_{1} x_{2}\right]=\xi^{-1} x_{2}^{-1} x_{1}^{-1} \xi x_{1} x_{2}=\xi^{-1} x_{1}^{-1} x_{2}^{-1} \xi x_{2} x_{1}=\xi^{-1} x_{1}^{-1} \xi\left[\xi, x_{2}\right] x_{1}=\left[\xi, x_{1}\right]\left[\xi, x_{2}\right]$, where the second equality follows from Lemma 2.9 and $\mathbb{N}_{r}^{a} / \mathbb{N}_{r}^{a+1}$ being abelian, and the fourth follows from Lemma 2.9 as $\left[\xi, x_{2}\right] \in \breve{G}_{r}^{r-1}$, the assumption $a \geq 2$, and the subsequent fact that $\mathbb{N}_{a+1}^{a}$ is generated by root subgroups contained in it. The linearity in $\bar{\xi}$ is shown similarly.

(ii): We work on $\overline{\mathbb{F}}_{q}$-points. To show the first claim, we observe that $\mathbb{U}_{\alpha, r}^{r-1}$ commutes with $\mathbb{N}_{r}^{2}$ by Lemma 2.9. As $\mathbb{N}_{r}^{1, i+1}$ is generated by $\mathbb{N}_{r}^{2}$ along with $\mathbb{U}_{\beta, r}^{1}$ for all $\beta$ which are either reductive or satisfy $\varepsilon_{\beta} \geq \varepsilon_{i}$, we have to show that $\left[\mathbb{U}_{\alpha, r}^{r-1}, \mathbb{U}_{\beta, r}^{1}\right] \subseteq \mathbb{G}_{r}^{r-1, s+1}$ for all such $\beta$. We have two cases:

Case ( $\beta$ is non-reductive). We have to show that $\left[\breve{U}_{\alpha, m_{\alpha}+r-2}, \breve{U}_{\beta, m_{\beta}}\right]$ maps to $\breve{G}_{r}^{r-1, s+1}$ inside $\breve{G}_{r}$. Using Lemma 2.8 (ii), it is enough to show that for all $p, q \in \mathbb{Z}_{\geq 1}$ such that $p \alpha+q \beta \in \Phi, \breve{U}_{p \alpha+q \beta, p\left(m_{\alpha}+r-2\right)+q m_{\beta}}$ maps to 1 in $\breve{G}_{r}$ if $p \alpha+q \beta$ is nonreductive and maps to $\breve{U}_{p \alpha+q \beta, r}^{r-1}$ if $p \alpha+q \beta$ is reductive. In both cases, this amounts to the claim that

$$
p\left(m_{\alpha}+r-2\right)+q m_{\beta} \geq m_{p \alpha+q \beta}+r-1,
$$

which in turn by Lemma 2.6 is equivalent to

$$
\left\lfloor p \varepsilon_{\alpha}+q \varepsilon_{\beta}\right\rfloor+(p-1)(r-2) \geq 1,
$$

which is true as $\varepsilon_{\beta} \geq \varepsilon_{i}=\varepsilon_{-\alpha}=1-\varepsilon_{\alpha}$. 
Case ( $\beta$ is reductive). This case is shown similarly (in fact, slightly simplier) to the above, and we omit the details. This finishes the proof of the first claim, i.e., $\left[\mathbb{U}_{\alpha, r}^{r-1}, \mathbb{N}_{r}^{1, i}\right] \subseteq \mathbb{G}_{r}^{r-1, s+1}$.

We now show the second claim, i.e., $\left[\mathbb{U}_{\alpha, r}^{r-1}, \mathbb{N}_{r}^{1, i+1}\right]=1$. Proceeding analogously as in the proof of the first claim, we need only to show that for all $\beta \in \Phi$ either reductive or satisfying $\varepsilon_{\beta} \geq \varepsilon_{i+1}$, one has $\left[\mathbb{U}_{\alpha, r}^{r-1}, \mathbb{U}_{\beta, r}^{1}\right]=1$. We again have two cases:

Case ( $\beta$ is non-reductive). We have to show that $\left[\breve{U}_{\alpha, m_{\alpha}+r-2}, \breve{U}_{\beta, m_{\beta}}\right]$ maps to 1 in $\breve{G}_{r}$. Using Lemma 2.8(ii), it is enough to show that for all $p, q \in \mathbb{Z}_{\geq 1}$ such that $p \alpha+q \beta \in \Phi, \breve{U}_{p \alpha+q \beta, p\left(m_{\alpha}+r-2\right)+q m_{\beta}}$ maps to 1 in $\breve{G}_{r}$. If $p \alpha+q \beta$ is non-reductive, this follows from the similar statement in the proof of the first claim, as $\varepsilon_{i+1} \geq \varepsilon_{i}$. If $p \alpha+q \beta$ is reductive, it amounts to claim that

$$
p\left(m_{\alpha}+r-2\right)+q m_{\beta} \geq m_{p \alpha+q \beta}+r,
$$

which by Lemma 2.6 is equivalent to

$$
\left\lfloor p \varepsilon_{\alpha}+q \varepsilon_{\beta}\right\rfloor+(p-1)(r-2) \geq 2,
$$

But this is true, as $\left\lfloor p \varepsilon_{\alpha}+q \varepsilon_{\beta}\right\rfloor \geq 2$. Indeed, as $p \alpha+q \beta$ is reductive, $\varepsilon_{p \alpha+q \beta}=0$. Hence by Lemma 2.6 $\left\lfloor p \varepsilon_{\alpha}+q \varepsilon_{\beta}\right\rfloor=p \varepsilon_{\alpha}+q \varepsilon_{\beta} \geq \varepsilon_{\alpha}+\varepsilon_{\beta}>1$. Being an integer, $\left\lfloor p \varepsilon_{\alpha}+q \varepsilon_{\beta}\right\rfloor$ must be $\geq 2$.

Case ( $\beta$ is reductive). This case is shown similarly (in fact, slightly simpler) to the above, and we omit the details. This finishes the proof of the second claim.

We are now ready to show that the claimed pairing is well-defined. Indeed, let $\xi \in \breve{U}_{\alpha, r}^{r-1}$ and let $x, x^{\prime} \in \breve{N}_{r}^{1, i}$ with the same image $\bar{x}=\bar{x}^{\prime} \in \breve{N}_{r}^{1, i} / \breve{N}_{r}^{1, i+1}$. Then there is an $y \in \breve{N}_{r}^{1, i+1}$ such that $x^{\prime}=x y$. We compute:

$$
\left[\xi, x^{\prime}\right]=[\xi, x y]=\xi^{-1} y^{-1} x^{-1} \xi x y=y^{-1}[\xi, x] y=[\xi, x],
$$

where for the third equality we use that $\left[\mathbb{U}_{\alpha, r}^{r-1}, \mathbb{N}_{r}^{1, i+1}\right]=1$ and for the last we use that $[\xi, x] \in \breve{G}_{r}^{r-1, s+1}$ and $\left[\mathbb{G}_{r}^{r-1, s+1}, \mathbb{N}_{r}^{1}\right]=1$ (indeed, for any reductive root $\gamma$ we have $\left[\mathbb{U}_{\gamma, r}^{r-1}, \mathbb{N}_{r}^{1}\right]=1$ by Lemma 2.9). Now we show that this pairing is linear in the second variable. Therefore, let $\xi \in \breve{U}_{\alpha, r}^{r-1}$ and $x_{1}, x_{2} \in \breve{N}_{r}^{1, i}$. We compute:

$$
\begin{aligned}
{\left[\xi, x_{1} x_{2}\right] } & =\xi^{-1} x_{2}^{-1} x_{1}^{-1} \xi x_{1} x_{2}=\xi^{-1}\left[x_{2}, x_{1}\right] x_{1}^{-1} x_{2}^{-1} \xi x_{1} x_{2} \\
& =\left[x_{2}, x_{1}\right] \xi^{-1} x_{1}^{-1} x_{2}^{-1} \xi x_{1} x_{2}=\left[x_{2}, x_{1}\right] \xi^{-1} x_{1}^{-1} x_{2}^{-1} \xi x_{2} x_{1}\left[x_{1}, x_{2}\right] \\
& =\left[x_{2}, x_{1}\right] \xi^{-1} x_{1}^{-1} \xi\left[\xi, x_{2}\right] x_{1}\left[x_{1}, x_{2}\right]=\left[x_{2}, x_{1}\right]\left[\xi, x_{1}\right]\left[\xi, x_{2}\right]\left[x_{1}, x_{2}\right] \\
& =\left[\xi, x_{1}\right]\left[\xi, x_{2}\right] .
\end{aligned}
$$

The third equality follows as $\left[x_{2}, x_{1}\right] \in \breve{N}_{r}^{1, i+1}$ (as $\mathbb{N}_{r}^{1, i} / \mathbb{N}_{r}^{1, i+1}$ is abelian) and as $\left[\mathbb{U}_{\alpha, r}^{r-1}, \mathbb{N}_{r}^{1, i+1}\right]=1$. The sixth equality follows as $\left[\xi, x_{2}\right] \in \breve{G}_{r}^{r-1, s+1}$ commutes with $x_{1} \in \breve{N}_{r}^{1}$. The last equality follows as $\left[\xi, x_{1}\right],\left[\xi, x_{2}\right] \in \breve{G}_{r}^{r-1, \mathrm{~s}+1}$ commute with $\left[x_{1}, x_{2}\right] \in \breve{N}_{r}^{1}$, and as $\left[x_{2}, x_{1}\right]\left[x_{1}, x_{2}\right]=1$. An analogous (slightly simplier) computation shows the linearity in the first variable.

Remark 4.6. Lemma 2.8(ii) can certainly be generalized. As we will not use the following generalization, we state it without proof. As for any root $\alpha \in \Phi,-\alpha$ is a root too, and $\varepsilon_{-\alpha}=1-\varepsilon_{\alpha}$, we have a symmetry between the jumps $\varepsilon_{i}$. Concretely, we have $\varepsilon_{i}=1-\varepsilon_{s+1-i}$ for $1 \leq i \leq s$. For each $1 \leq a \leq r-1$, let $\mathbb{G}_{r}^{a, i}$ be 
the subgroup of $\mathbb{G}_{r}^{a}$ generated by $\mathbb{G}_{r}^{a+1}, \mathbb{T}_{r}^{a}, \mathbb{U}_{\alpha, r}^{a}\left(\alpha\right.$ reductive or $\left.\varepsilon_{\alpha} \geq \varepsilon_{i}\right)$. Then Lemma 2.8 extends to the following general duality statement: Fix $1 \leq a \leq r-1$ and $1 \leq i \leq s$. Then the commutator induces a bilinear pairing,

$$
\mathbb{G}_{r}^{r-a, s+1-i} / \mathbb{G}_{r}^{r-a, s+2-i} \times \mathbb{G}_{r}^{a, i} / \mathbb{G}_{r}^{a, i+1} \rightarrow \mathbb{G}_{r}^{r-1, s+1} .
$$

4.5. Stratification on (subgroups of) $\mathbb{N}_{r}^{1}$. Recall that for any subgroup $H \subset G$ and associated subgroups $\mathbb{H}_{r} \subset \mathbb{G}_{r}$, we have the notation $\mathbb{H}_{r}^{a, *}=\mathbb{H}_{r}^{a} \backslash \mathbb{H}_{r}^{a+1}$ (open subscheme) and hence the corresponding set $\breve{H}_{r}^{a, *}$ of $\overline{\mathbb{F}}_{q}$-valued points.

Lemma 4.7. Let $r \geq 2$ and let $r-1 \geq a \geq 1$. For $z \in \breve{N}_{r}^{a, *}$, write $z=\prod_{\beta \in \Phi^{+}} x_{\beta}^{z}$ with $x_{\beta}^{z} \in \breve{U}_{\beta, r}^{a}$ for a fixed (but arbitrary) order on $\Phi^{+}$. For $\beta \in \Phi^{+}$, let $a \leq$ $a(\beta, z) \leq r$ be the integer such that $x_{\beta}^{z} \in \breve{U}_{\beta, r}^{a(\beta, z), *}$.

(i) If $a \geq 2$, then the set

$$
A_{z}:=\left\{\beta \in \Phi^{+}: a(\beta, z)=a\right\}
$$

is non-empty and independent of the chosen order on $\Phi^{+}$.

(ii) Let $a=1$ and let $s+1 \geq i \geq 1$ be such that $z \in \breve{N}_{r}^{1, i, *}$. Then the set

$$
A_{z}:=\left\{\beta \in \Phi_{i}^{+}: a(\beta, z)=1\right\}
$$

is non-empty and independent of the chosen order on $\Phi^{+}$. Moreover, $a(\beta, z)$ $>1$ for all $\beta \in \bigcup_{j=1}^{i-1} \Phi_{j}^{+}$.

Proof.

(i): As $a \geq 2$, the quotient $\mathbb{N}_{r}^{a} / \mathbb{N}_{r}^{a+1}$ is abelian by Lemma 4.4. Thus its $\overline{\mathbb{F}}_{q}$-points are simply tuples $\left(\bar{x}_{\beta}\right)_{\beta \in \Phi^{+}}$with $\bar{x}_{\beta} \in \breve{U}_{\beta, a+1}^{a}$ with entry-wise multiplication. If $\bar{z}=\left(\bar{x}_{\beta}^{z}\right)$ is the image of $z$ in this quotient, then $A_{z}$ identifies with the set of those $\beta$ for which $\bar{x}_{\beta}^{z} \neq 1$ (which is obviously independent of the order).

(ii): Assume that the last claim of (ii) is not true. Then let $1 \leq i_{0}<i$ be the smallest integer such that $a(\beta, z)=1$ for some $\beta \in \Phi_{i_{0}}^{+}$. Then from Lemma 4.4 it follows that $z \in \breve{N}_{r}^{1, i_{0}, *}$, which contradicts the assumption. This shows the last claim. The first claim follows by the same argument as in (i).

Using Section 4.4 we can now prove the following generalization of Lus04, Lemma 1.7].

Definition 4.8. For $\alpha \in \Phi^{+}$define its height ht $(\alpha)$ (relative to $N$ ) to be the largest integer $m \geq 1$ such that $\alpha=\sum_{i=1}^{m} \alpha_{i}$ with $\alpha_{i} \in \Phi^{+}$.

Proposition 4.9. Let $r \geq 2$ and let $r-1 \geq a \geq 1$. Let $z=\prod_{\beta \in \Phi^{+}} x_{\beta}^{z} \in \breve{N}_{r}^{a, *}$ for $x_{\beta}^{z} \in \breve{U}_{\beta, r}^{a}$ and let $A_{z}$ be as in Lemma 4.7.

(i) If $A_{z}$ contains a non-reductive root, let $-\alpha \in A_{z}$ be a non-reductive root of maximal height and $\alpha \in \Phi^{-}$its opposite. Then for any $\xi \in \mathbb{U}_{\alpha, r}^{r-a}$, we have $[\xi, z] \in \mathcal{T}^{\alpha} \mathbb{N}_{r}^{-, r-1}$. Moreover, projecting $[\xi, z]$ into $\mathcal{T}^{\alpha}$ induces an isomorphism

$$
\lambda_{z}: \mathbb{U}_{\alpha, r}^{r-a} / \mathbb{U}_{\alpha, r}^{r-a+1} \stackrel{\sim}{\rightarrow} \mathcal{T}^{\alpha}
$$

(ii) If $A_{z}$ contains only reductive roots, let $-\alpha \in A_{z}$ be a root of maximal height and $\alpha \in \Phi^{-}$its opposite. Then for any $\xi \in \mathbb{U}_{\alpha, r}^{r-a-1}$, we have $[\xi, z] \in$ $\mathcal{T}^{\alpha} \mathbb{N}_{r}^{-, r-1}$. Moreover, projecting $[\xi, z]$ into $\mathcal{T}^{\alpha}$ induces an isomorphism

$$
\lambda_{z}: \mathbb{U}_{\alpha, r}^{r-a-1} / \mathbb{U}_{\alpha, r}^{r-a} \stackrel{\sim}{\rightarrow} \mathcal{T}^{\alpha}
$$


Proof. Parts (i) and (ii) can be proven in the same way. We give the full proof of (i) only.

Proof of (i) when $a \geq 2$. We work on $\overline{\mathbb{F}}_{q}$-points. Assume that $A_{z}$ contains a nonreductive root and let $-\alpha$ be such a root of maximal height and $\alpha \in \Phi^{-}$its opposite. Let $\xi \in \breve{U}_{\alpha, r}^{r-a}$ and let $\bar{\xi} \in \breve{U}_{\alpha, r}^{r-a} / \breve{U}_{\alpha, r}^{r-a+1}$ and $\bar{z} \in \breve{N}_{r}^{a} / \breve{N}_{r}^{a+1}$ be the images of $\xi$ and $z$ respectively. By Lemma 4.4 we may write

$$
\bar{z}=\bar{x}_{-\alpha}^{z} \prod_{\beta \in \Phi^{+} \text {red. }} \bar{x}_{\beta}^{z} \cdot \prod_{\substack{\beta \in \Phi^{+} \text {non-red., } \beta \neq-\alpha \\ \text { ht }(\beta) \leq \text { ht }(-\alpha)}} \bar{x}_{\beta}^{z},
$$

where $\bar{x}_{\beta}^{z} \in \breve{U}_{\beta, r}^{a} / \breve{U}_{\beta, r}^{a+1}$ and where the products are taken in any order. Lemma 4.5 shows that $[\xi, z]$ is the product of $\left[\bar{\xi}, \bar{x}_{-\alpha}^{z}\right]$ with all the $\left[\bar{\xi}, \bar{x}_{\beta}^{z}\right]$ for $\beta \in \Phi^{+}$, the product taken in any order. If $\beta$ is reductive, then $\left[\bar{\xi}, \bar{x}_{\beta}^{z}\right] \in\left[\breve{U}_{\alpha, r}^{r-a}, \breve{U}_{\beta, r}^{a}\right]=1$ by Lemma 2.9. If $\beta \neq-\alpha$ is non-reductive, then by assumption $\operatorname{ht}(\beta) \leq \operatorname{ht}(-\alpha)$. The commutator $\left[\bar{\xi}, \bar{x}_{\beta}^{z}\right]$ is the image of an element of

$$
\left[\breve{U}_{\alpha, m_{\alpha}+(r-a)-1}, \breve{U}_{\beta, m_{\beta}+a-1}\right] \subseteq \prod_{\substack{p, q \in \mathbb{Z}_{\geq 1} \\ p \alpha+q \beta \in \Phi}} \breve{U}_{p \alpha+q \beta, p m_{\alpha}+q m_{\beta}+p(r-a-1)+q(a-1)}
$$

Lemma 4.10. The image of the right hand side of (4.4) in $\breve{G}_{r}$ lies in $\breve{N}_{r}^{-, r-1}$.

Proof. It is enough to show that for each $(p, q)$ occurring in the product, the corresponding factor is either contained in $\breve{N}_{r}^{-, r-1}$ or vanishes in $\breve{G}_{r}$. If $p \geq q$, then ht $(\beta) \leq \operatorname{ht}(-\alpha)$ implies $p \alpha+q \beta \notin \Phi^{+}$. So, we may assume that $q>p$ and in particular $q \geq 2$. It is enough to show that

$$
\breve{U}_{p \alpha+q \beta, p m_{\alpha}+q m_{\beta}+p(r-a-1)+q(a-1)} \subseteq \begin{cases}\breve{U}_{p \alpha+q \beta, m_{p \alpha+q \beta}+r} & \text { if } p \alpha+q \beta \text { reductive } \\ \breve{U}_{p \alpha+q \beta, m_{p \alpha+q \beta}+r-1} & \text { otherwise }\end{cases}
$$

as both map to 1 in $\breve{G}_{r}$. Equivalently, we have to show that

$p m_{\alpha}+q m_{\beta}-m_{p \alpha+q \beta}+p(r-a-1)+q(a-1)-(r-1) \geq \begin{cases}1 & \text { if } p \alpha+q \beta \text { reductive } \\ 0 & \text { otherwise. }\end{cases}$

But this holds as by Lemma 2.6 $p m_{\alpha}+q m_{\beta}-m_{p \alpha+q \beta}=\left\lfloor p \varepsilon_{\alpha}+q \varepsilon_{\beta}\right\rfloor$ is $\geq 1$ if $p \alpha+q \beta$ is reductive and is $\geq 0$ otherwise, and as $q \geq 2$ and $a \geq 2$.

Finally, $\left[\bar{\xi}, \bar{x}_{-\alpha}^{z}\right]=\left[\xi, x_{-\alpha}^{z}\right] \in \mathcal{T}^{\alpha}\left(\overline{\mathbb{F}}_{q}\right)$ by Lemma $2.8($ iii). Thus $[\xi, z] \in$ $\mathcal{T}^{\alpha}\left(\overline{\mathbb{F}}_{q}\right) \breve{N}_{r}^{-, r-1}$. Moreover, if we project onto $\mathcal{T}^{\alpha}\left(\overline{\mathbb{F}}_{q}\right)$, then only $\left[\bar{\xi}, \bar{x}_{-\alpha}^{z}\right]$ survives and Lemma 2.8(iii) proves the desired isomorphism $\lambda_{z}$. This finishes the proof of (i) in the case $a \geq 2$.

Proof of (i) when $a=1$. Let $s \geq i \geq 1$ denote the integer such that $z \in \breve{N}^{1, i, *}$. (Note that $i \neq s+1$ as $A_{z}$ contains a non-reductive root by assumption). We have $\xi \in \breve{U}_{\alpha, r}^{r-1}$, and we let $\bar{z}$ denote the image of $z$ in $\breve{N}_{r}^{1, i} / \breve{N}_{r}^{1, i+1}$. By Lemma 4.4 we may write

$$
\bar{z}=\bar{x}_{-\alpha}^{z}\left(\prod_{\substack{\beta \in \Phi_{i}^{+}: \beta \neq-\alpha \\ \operatorname{ht}(\beta) \leq \mathrm{ht}(-\alpha)}} \bar{x}_{\beta}^{z}\right),
$$


(product are taken in any order). By Lemma 4.5, $[\xi, \bar{z}]$ is the product of $\left[\xi, \bar{x}_{-\alpha}^{z}\right]$ with all the $\left[\xi, \bar{x}_{\beta}^{z}\right]$ taken in any order. By assumption $\varepsilon_{\beta}=\varepsilon_{i}=\varepsilon_{-\alpha}=1-\varepsilon_{\alpha}$. In particular, all $\beta$ 's are non-reductive. Now, $\left[\xi, \bar{x}_{\beta}^{z}\right]$ is the image in $\breve{G}_{r}^{r-1, s+1}$ of an element of

$$
\left[\breve{U}_{\alpha, m_{\alpha}+r-2}, \breve{U}_{\beta, m_{\beta}}\right] \subseteq \prod_{\substack{p, q \in \mathbb{Z}_{\geq 1} \\ p \alpha+q \beta \in \Phi}} \breve{U}_{p \alpha+q \beta, p m_{\alpha}+q m_{\beta}+p(r-2)}
$$

Lemma 4.11. The image of the right hand side of (4.5) in $\breve{G}_{r}$ lies in $\breve{N}_{r}^{-, r-1}$.

Proof. Note that the right hand side of (4.5) is contained in $\breve{G}_{r}^{r-1, s+1}$ (exactly as in the proof of Lemma 4.5(ii)). Now the same arguments as in the proof Lemma 4.10 apply. If $p \geq q$, then ht $(\beta) \leq$ ht $(-\alpha)$ implies $p \alpha+q \beta \notin \Phi^{+}$, thus the corresponding factor of the product is contained in $\breve{N}_{r}^{-} \cap \breve{G}_{r}^{r-1, s+1} \subseteq \breve{N}_{r}^{-,}, r-1$. Thus we may assume that $q>p$ and in particular $q \geq 2$. It is enough to show that

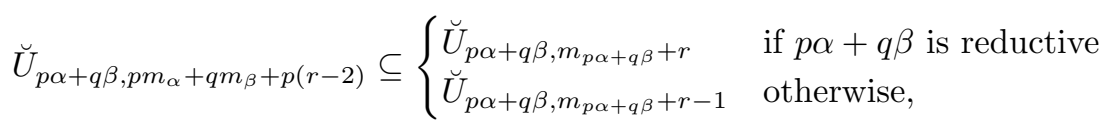

as both map to 1 in $\breve{G}_{r}$. Equivalently, we have to show that

$$
p m_{\alpha}+q m_{\beta}-m_{p \alpha+q \beta}+p(r-2)-(r-1) \geq \begin{cases}1 & \text { if } p \alpha+q \beta \text { is reductive } \\ 0 & \text { otherwise. }\end{cases}
$$

By Lemma 2.6. this follows from $\left\lfloor p \varepsilon_{\alpha}+q \varepsilon_{\beta}\right\rfloor \geq 2$ if $p \alpha+q \beta$ is reductive, resp. to $\left\lfloor p \varepsilon_{\alpha}+q \varepsilon_{\beta}\right\rfloor \geq 1$ if $p \alpha+q \beta$ is non-reductive. But in any case we have $p \varepsilon_{\alpha}+q \varepsilon_{\beta} \geq$ $\varepsilon_{\alpha}+2\left(1-\varepsilon_{\alpha}\right)=2-\varepsilon_{\alpha}>1$ by assumptions. In particular, we are done in the case when $p \alpha+q \beta$ is non-reductive. If $p \alpha+q \beta$ is reductive, then $p \varepsilon_{\alpha}+q \varepsilon_{\beta}$ must also be an integer (by Lemma 2.6) and hence $\geq 2$, and we are done in this case too.

Finally, $\left[\xi, \bar{x}_{-\alpha}^{z}\right] \in \mathcal{T}^{\alpha}\left(\overline{\mathbb{F}}_{q}\right)$ by Lemma 2.8(iii). Thus $[\xi, z] \in \mathcal{T}^{\alpha}\left(\overline{\mathbb{F}}_{q}\right) \breve{N}_{r}^{-, r-1}$. Moreover, if we project onto $\mathcal{T}^{\alpha}\left(\overline{\mathbb{F}}_{q}\right)$, then only $\left[\bar{\xi}, \bar{x}_{-\alpha}^{z}\right]$ survives and Lemma 2.8(iii) proves the desired isomorphism $\lambda_{z}$. This finishes the proof of (i).

Remark 4.12. We note that in the proof of [Lus04, Lemma 1.7] there is an (easily correctable) mistake. It is claimed that whenever $-\alpha, \beta \in \Phi^{+}$with $-\alpha \neq \beta$ and ht $(-\alpha) \geq \operatorname{ht}(\beta)$, then $p \alpha+q \beta \notin \Phi^{+}$for all $p, q \in \mathbb{Z}_{\geq 1}$. This is not true. For example, let $\Phi$ be of type $C_{2}$, let $\epsilon_{1}, \epsilon_{2}$ denote a basis for $X^{*}(T)$ such that the $\Phi^{+}=\left\{\epsilon_{1}-\epsilon_{2}, \epsilon_{1}+\epsilon_{2}, 2 \epsilon_{1}, 2 \epsilon_{2}\right\}$. Then taking $\alpha=-2 \epsilon_{1}, \beta=\epsilon_{1}+\epsilon_{2}$. Then $\operatorname{ht}(-\alpha)=$ $3>2=\operatorname{ht}(\beta)$. But $\alpha+2 \beta=2 \epsilon_{2} \in \Phi^{+}$. Observe here that $\alpha+\beta \notin \Phi^{+}$, which contradicts the parenthetical assertion at the end of the proof of Lus04, Lemma 1.7].

Surely, the statement of [Lus04, Lemma 1.7] remains true. The place in its proof, where the abovementioned claim is used, can be corrected as follows: if $p \alpha+q \beta \in \Phi^{+}$for some $p, q \in \mathbb{Z}_{\geq 1}$, then $q \geq 2$ and the part of the commutator (as in the proof of Proposition 4.9) inside $\mathbb{U}_{p \alpha+q \beta, r}$ vanishes, since all roots are reductive and $r \geq 2$. 
Let $\mathbb{K}_{r}=\mathbb{U}_{r}^{-} \cap \mathbb{N}_{r}$. Let $\Phi^{\prime}=\left\{\beta \in \Phi^{+}: \mathbb{U}_{\beta, r} \subseteq \mathbb{K}_{r}\right\}$. Let $\mathcal{X}$ denote the set of all non-empty subsets $I \subseteq \Phi^{\prime}$ satisfying

(i) the restriction of ht: $\Phi^{+} \rightarrow \mathbb{Z}_{\geq 0}$ to $I$ is constant, and

(ii) $I$ contains either only reductive or only non-reductive roots.

To $z \in \breve{K}_{r}^{1} \backslash\{1\}$ we attach a pair $\left(a_{z}, I_{z}\right)$ with $1 \leq a_{z} \leq r-1$ and $I_{z} \in \mathcal{X}$. Define $a_{z}$ by $z \in \breve{K}_{r}^{a_{z}, *}$. Let $A_{z}$ be as in Lemma 4.7. If $A_{z}$ contains a non-reductive root, let $I_{z} \subseteq A_{z}$ be the subset of all non-reductive roots of maximal height. If $A_{z}$ contains only reductive roots, let $I_{z} \subseteq A_{z}$ be the subset of all roots of maximal height. We have a stratification into locally closed subsets

$$
\mathbb{K}_{r}^{1} \backslash\{1\}=\bigsqcup_{a, I} \mathbb{K}_{r}^{a, *, I}, \quad \text { where } \mathbb{K}_{r}^{a, *, I}\left(\overline{\mathbb{F}}_{q}\right)=\left\{z \in \breve{K}_{r}^{1} \backslash\{1\}:\left(a_{z}, I_{z}\right)=(a, I)\right\} .
$$

4.6. Cohomology of $\widehat{\Sigma}^{\prime}$. We now prove Proposition 4.2. Using the stratification (4.6) and Proposition 4.9, the proof of Proposition 4.2 is very similar to the proof of Lus04, 1.9 (c)]. We sketch the arguments here. It is enough to show that $H_{c}^{j}\left(\widehat{\Sigma}_{w}^{\prime}\right)_{\theta, \theta^{\prime}}=0$ for all $j \geq 0$. For a $\mathcal{T}^{\prime}\left(\overline{\mathbb{F}}_{q}\right)^{\sigma}$-module $M$ and a character $\chi$ of $\mathcal{T}^{\prime}\left(\overline{\mathbb{F}}_{q}\right)^{\sigma}$, write $M_{(\chi)}$ for the $\chi$-isotypic component of $M$. Note that $\mathcal{T}^{\prime}\left(\overline{\mathbb{F}}_{q}\right)^{\sigma}$ acts on $\widehat{\Sigma}_{w}^{\prime}$ by

$$
t^{\prime}:\left(x, x^{\prime}, u, u^{\prime}, z, \tau^{\prime}\right) \mapsto\left(x, t^{\prime} x^{\prime} t^{\prime-1}, u, t^{\prime} u^{\prime} t^{\prime-1}, z, \tau^{\prime} t^{\prime-1}\right) .
$$

Hence $H_{c}^{j}\left(\widehat{\Sigma}_{w}^{\prime}\right)$ is a $\mathcal{T}^{\prime}\left(\mathbb{F}_{q}\right)$-module. It is enough to show that $H_{c}^{j}\left(\widehat{\Sigma}_{w}^{\prime}\right)_{(\chi)}=0$ for any regular character $\chi$ of $\mathcal{T}^{\prime}\left(\mathbb{F}_{q}\right)$. Fix such a $\chi$. Set $N=\dot{w} U^{\prime-} \dot{w}^{-1}, N^{-}=\dot{w} U^{\prime} \dot{w}^{-1}$. The stratification (4.6) of $\mathbb{K}_{r}^{1} \backslash\{1\}$ induces a stratification of $\widehat{\Sigma}_{w}^{\prime}$ into locally closed subsets indexed by $1 \leq a \leq r-1$ and $I \in \mathcal{X}$ :

$$
\widehat{\Sigma}_{w}^{\prime}=\bigsqcup_{a, I} \widehat{\Sigma}_{w}^{\prime, a, I} \quad \text { where } \widehat{\Sigma}_{w}^{\prime, a, I}\left(\overline{\mathbb{F}}_{q}\right)=\left\{\left(x, x^{\prime}, u, u^{\prime}, z, \tau^{\prime}\right) \in \widehat{\Sigma}_{w}^{\prime}\left(\overline{\mathbb{F}}_{q}\right): z \in \breve{K}_{r}^{a, *, I}\right\} .
$$

Note that each $\widehat{\Sigma}_{w}^{\prime, a, I}$ is stable under $\mathcal{T}^{\prime}\left(\mathbb{F}_{q}\right)$. Thus (4.2) follows from

$$
H_{c}^{j}\left(\widehat{\Sigma}_{w}^{\prime, a, I}, \overline{\mathbb{Q}}_{\ell}\right)_{(\chi)}=0 \quad \text { for any fixed } a, I .
$$

To show (4.7), choose a root $\alpha$ such that $-\alpha \in I$. Then $\mathbb{U}_{\alpha, r} \subseteq \mathbb{U}_{r} \cap \dot{w} \mathbb{U}_{r}^{\prime} \dot{w}^{-1}$. By Proposition 4.9 for any $z \in \breve{K}_{r}^{a, *, I}$, we have an isomorphism

$$
\begin{array}{ll}
\lambda_{z}: \mathbb{U}_{\alpha, r}^{r-a} / \mathbb{U}_{\alpha, r}^{r-a+1} \stackrel{\sim}{\longrightarrow} \mathcal{T}^{\alpha}, & \text { if } \alpha \text { is non-reductive, } \\
\lambda_{z}: \mathbb{U}_{\alpha, r}^{r-a-1} / \mathbb{U}_{\alpha, r}^{r-a} \stackrel{\sim}{\longrightarrow} \mathcal{T}^{\alpha}, & \text { if } \alpha \text { is reductive. }
\end{array}
$$

Let $\pi$ denote the natural projection $\mathbb{U}_{\alpha, r}^{r-a} \rightarrow \mathbb{U}_{\alpha, r}^{r-a} / \mathbb{U}_{\alpha, r}^{r-a+1}$ if $\alpha$ is non-reductive and the natural projection $\mathbb{U}_{\alpha, r}^{r-a-1} \rightarrow \mathbb{U}_{\alpha, r}^{r-a-1} / \mathbb{U}_{\alpha, r}^{r-a}$ if $\alpha$ is reductive. Let $\psi$ be a section to $\pi$ such that $\pi \psi=1$ and $\psi(1)=1$. Let

$$
\mathcal{H}^{\prime}:=\left\{t^{\prime} \in \mathcal{T}^{\prime}: t^{\prime-1} \sigma\left(t^{\prime}\right) \in \dot{w}^{-1} \mathcal{T}^{\alpha} \dot{w}\right\}
$$

This is a closed subgroup of $\mathcal{T}^{\prime}$. For any $t^{\prime} \in \mathcal{T}^{\prime}$ define $f_{t^{\prime}}: \widehat{\Sigma}_{w}^{\prime, a, I} \rightarrow \widehat{\Sigma}_{w}^{\prime, a, I}$ by

$$
f_{t^{\prime}}\left(x, x^{\prime}, u, u^{\prime}, z, \tau^{\prime}\right)=\left(x \sigma(\xi), \hat{x}^{\prime}, u, \sigma\left(t^{\prime}\right)^{-1} u^{\prime} \sigma\left(t^{\prime}\right), z, \tau^{\prime} \sigma\left(t^{\prime}\right)\right),
$$

where

$$
\xi=\psi \lambda_{z}^{-1}\left(\dot{w} \sigma\left(t^{\prime}\right)^{-1} t^{\prime} \dot{w}^{-1}\right) \in \begin{cases}\mathbb{U}_{\alpha, r}^{r-a-1} \subseteq \mathbb{U}_{r} \cap \dot{w} \mathbb{U}_{r}^{\prime} \dot{w}^{-1} & \text { if } \alpha \text { is reductive } \\ \mathbb{U}_{\alpha, r}^{r-a} \subseteq \mathbb{U}_{r} \cap \dot{w} \mathbb{U}_{r}^{\prime} \dot{w}^{-1} & \text { otherwise }\end{cases}
$$


and $\hat{x}^{\prime} \in \mathbb{G}_{r}$ is defined by the condition that

$$
x \sigma\left(\xi z \dot{w} \tau^{\prime} \sigma\left(t^{\prime}\right)\right) \in u z \dot{w} \tau^{\prime} \sigma\left(t^{\prime}\right) \sigma\left(t^{\prime}\right)^{-1} u^{\prime} \sigma\left(t^{\prime}\right) \hat{x}^{\prime} .
$$

To check that $f_{t^{\prime}}$ is well-defined we have to show $\hat{x}^{\prime} \in \sigma\left(\mathbb{U}_{r}^{\prime}\right)$. This is done with exactly the same computation as in the proof of [Lus04, Lemma 1.9], and we omit this. It is clear that $f_{t^{\prime}}: \widehat{\Sigma}_{w}^{\prime, a, I} \rightarrow \widehat{\Sigma}_{w}^{\prime, a, I}$ is an isomorphism for any $t^{\prime} \in \mathcal{H}^{\prime}$. Moreover, since $\mathcal{T}^{\prime}\left(\mathbb{F}_{q}\right) \subseteq \mathcal{H}^{\prime}$ and since for any $t^{\prime} \in \mathcal{T}^{\prime}\left(\mathbb{F}_{q}\right)$ the map $f_{t^{\prime}}$ coincides with the action of $t^{\prime}$ in the $\mathcal{T}^{\prime}\left(\mathbb{F}_{q}\right)$-action on $\widehat{\Sigma}_{w}^{\prime, a, I}$ (we use $\psi(1)=1$ here), it follows that we have constructed an action $f$ of $\mathcal{H}^{\prime}$ on $\widehat{\Sigma}_{w}^{\prime, a, I}$ extending the $\mathcal{T}^{\prime}\left(\mathbb{F}_{q}\right)$-action.

If a connected group acts on a scheme, the induced action in the cohomology is constant. Thus for any $t^{\prime} \in \mathcal{H}^{\prime \circ}$, the induced map $f_{t^{\prime}}^{*}: H_{c}^{j}\left(\widehat{\Sigma}_{w}^{\prime, a, I}, \overline{\mathbb{Q}}_{\ell}\right) \rightarrow$ $H_{c}^{j}\left(\widehat{\Sigma}_{w}^{\prime, a, I}, \overline{\mathbb{Q}}_{\ell}\right)$ is constant when $t^{\prime}$ varies in $\mathcal{H}^{\prime \circ}$. Hence $\mathcal{T}^{\prime}\left(\mathbb{F}_{q}\right) \cap \mathcal{H}^{\prime \circ}$ acts trivially on $H_{c}^{j}\left(\widehat{\Sigma}_{w}^{\prime, a, I}, \overline{\mathbb{Q}}_{\ell}\right)$.

We can find some $m \geq 1$ such that $\sigma^{m}\left(\dot{w}^{-1} \mathcal{T}^{\alpha} \dot{w}\right)=\dot{w}^{-1} \mathcal{T}^{\alpha} \dot{w}$. Then

$$
t^{\prime} \mapsto t^{\prime} \sigma\left(t^{\prime}\right) \sigma^{2}\left(t^{\prime}\right) \cdots \sigma^{m-1}\left(t^{\prime}\right)
$$

defines a morphism $\dot{w}^{-1} \mathcal{T}^{\alpha} \dot{w} \rightarrow \mathcal{H}^{\prime}$. Since $\mathcal{T}^{\alpha}$ is connected, its image is also connected and hence contained in $\mathcal{H}^{\prime \circ}$. If $t^{\prime} \in\left(\dot{w}^{-1} \mathcal{T}^{\alpha}\left(\overline{\mathbb{F}}_{q}\right) \dot{w}\right)^{\sigma^{m}}$, then $N_{\sigma}^{\sigma^{m}}\left(t^{\prime}\right) \in$ $\mathcal{T}^{\prime}\left(\overline{\mathbb{F}}_{q}\right)^{\sigma}$ and hence also $N_{\sigma}^{\sigma^{m}}\left(t^{\prime}\right) \in \mathcal{T}^{\prime}\left(\overline{\mathbb{F}}_{q}\right)^{\sigma} \cap \mathcal{H}^{\prime \circ}\left(\overline{\mathbb{F}}_{q}\right)$. Thus the action of $N_{\sigma}^{\sigma^{m}}\left(t^{\prime}\right) \in$ $\mathcal{T}^{\prime}\left(\overline{\mathbb{F}}_{q}\right)^{\sigma}$ on $H_{c}^{j}\left(\widehat{\Sigma}_{w}^{\prime, a, I}\right)$ is trivial for any $t^{\prime} \in\left(\dot{w}^{-1} \mathcal{T}^{\alpha}\left(\overline{\mathbb{F}}_{q}\right) \dot{w}\right)^{\sigma^{m}}$.

Finally, observe that if $H_{c}^{j}\left(\widehat{\Sigma}_{w}^{\prime, a, I}, \overline{\mathbb{Q}}_{\ell}\right)_{(\chi)} \neq 0$, then the above shows that $t^{\prime} \mapsto$ $\chi\left(N_{\sigma}^{\sigma^{m}}\left(t^{\prime}\right)\right)$ must be the trivial character, which contradicts the regularity assumption on $\chi$. This establishes (4.7), finishing the proof of Propositon 4.2

\section{TRACES OF VERY REgUlaR ELEMENTS}

Let the notation be as in the beginning of Section 4 The finale of this section is the proof of Theorem 1.2

Definition 5.1. We say that $s \in \breve{P}_{\mathbf{x}}$ is unramified very regular with respect to $\mathbf{x}$ if the following conditions hold:

(i) $s$ is a regular semisimple element of $G_{\breve{k}}$,

(ii) the connected centralizer $Z^{\circ}(s)$ of $s$ is a $\breve{k}$-split maximal torus of $G_{\breve{k}}$ whose apartment contains $\mathbf{x}$, and

(iii) $\alpha(s) \not \equiv 1$ modulo $\mathfrak{p}$ for all roots $\alpha$ of $Z^{\circ}(s)$ in $G_{\breve{k}}$.

For $r \geq 2$, we say that $s \in \mathbb{G}_{r}$ is unramified very regular, if $s$ is the image of an unramified very regular element of $\breve{P}_{\mathbf{x}}$.

Note that condition (ii) implies condition (i). Note that in condition (iii) the character $\alpha: Z^{\circ}(s) \rightarrow \mathbb{G}_{m, \breve{k}}$ induces a homomorphism of maximal bounded subgroups: $\alpha: \breve{Z}^{\circ}(s) \rightarrow \mathcal{O}^{\times}$, and hence the condition makes sense.

Remark 5.2. When $G$ is an inner form of $\mathrm{GL}_{n}$ and $T$ is the maximal nonsplit unramified torus in $G$, Definition 5.1 says that $x \in\left(\breve{T}^{0}\right)^{\sigma}=\mathcal{O}_{L}^{\times}$(here $\breve{k} \supseteq L \supseteq k$ is the degree- $n$-subextension) is unramified very regular if and only if the image of $x$ in $\left(\mathcal{O}_{L} / U_{L}^{1}\right) \cong \mathbb{F}_{q^{n}}^{\times}$has trivial $\operatorname{Gal}\left(\mathbb{F}_{q^{n}} / \mathbb{F}_{q}\right)$-stabilizer. This is not equivalent to (though is implied by) the condition that the image of $x$ in $\mathbb{F}_{q^{n}}^{\times}$is a generator although this last condition is sometimes also associated to the same terminology Hen92, BW13, CI20. 
Note that if $s \in \breve{P}_{\mathbf{x}}$ is unramified very regular, then we may consider the $W_{\mathbf{x}}(T)$ homogeneous space $W_{\mathbf{x}}\left(T, Z^{\circ}(s)\right.$ ) (see Section 2.8).

Before proving Theorem 1.2 , we point out the following corollary.

Corollary 5.3. Let $T^{\prime} \subset G$ be a $k$-rational $\breve{k}$-split maximal torus whose apartment contains $\mathbf{x}$. If $T$ and $T^{\prime}$ are not conjugate by an element of $\breve{P}_{\mathbf{x}}^{\sigma}$, then for any $s \in T^{\prime}(k)$ unramified very regular with respect to $\mathbf{x}$,

$$
\operatorname{Tr}\left(s, R_{T, U, r}^{\theta}\right)=0 .
$$

Proof. We need to show that for two such tori, $W_{\mathbf{x}}\left(T, T^{\prime}\right)^{\sigma}=\varnothing$. Suppose there is an element $w \in W_{\mathbf{x}}\left(T, T^{\prime}\right)^{\sigma}$. Then its preimage in $N_{\mathbb{G}_{r}}\left(\mathbb{T}_{r}, \mathbb{T}_{r}^{\prime}\right)$ form a $\mathbb{F}_{q}$-rational $\mathbb{T}_{r}$-torsor, which by Lang's theorem has a rational point. Doing this for all $r$ and using that the inverse limit of a family of non-empty compact sets is non-empty, we can find an element $n \in \breve{P}_{\mathbf{x}}^{\sigma}$, which conjugates $T(\mathcal{O})$ into $T^{\prime}(\mathcal{O})$. The centralizer of $T(\mathcal{O})$ in $G(\breve{k})$ is $T(\breve{k})$ (and similarly for $T^{\prime}$ ), so $n$ also conjugates $T(\breve{k})$ into $T^{\prime}(\breve{k})$, and so it conjugates $T$ into $T^{\prime}$, which contradicts the assumption.

We now make some preparations that we will use to prove Theorem 1.2. Let $B$ denote the Borel subgroup of $G$ whose unipotent radical is the fixed subgroup $U$, and let $\mathbb{B}_{r}$ be the corresponding subgroup of $\mathbb{G}_{r}$. The following result shows that $\mathbb{B}_{r}$ behaves in certain aspects like a Borel subgroup of $\mathbb{G}_{r}$ (although it is not a Borel subgroup if $r \geq 2$ ). Similar results in the case that $P_{\mathbf{x}}$ is reductive are shown in Sta12.

Proposition 5.4. Let $s \in \breve{G}_{r}$ be an unramified very regular element. If $x \in \breve{G}_{r}$ is such that $s \in x \breve{B}_{r} x^{-1}$, then there exists a unique $w \in W_{\mathbf{x}}\left(T, Z^{0}(s)\right)$ such that for any lift $\dot{w} \in \breve{G}_{r}$, we have $x \in \dot{w} \breve{B}_{r}$.

Proof. The maximal $\breve{k}$-split tori $T$ and $Z^{\circ}(s)$ are conjugate by an element $y \in \breve{P}_{\mathbf{x}}$, as $\mathbf{x}$ is contained in the intersection of their apartments. Conjugating by $y$ we thus may reduce to the special case that $Z^{\circ}(s)=T$.

We first prove the assertion in the case $r=1$. The image of $s$ in the reductive group $\breve{G}_{1}$ is regular semisimple and $\mathbb{B}_{1} \subseteq \mathbb{G}_{1}$ is a Borel subgroup. By DL76. Proposition $4.4(\mathrm{ii})$ ], we see that there is an element $\dot{w} \in \breve{G}_{1}$ normalizing $T$, and satisfying $x \mathbb{B}_{1} x^{-1}=\dot{w}^{-1} \mathbb{B}_{1} \dot{w}$. Since Borel subgroups are self-normalizing, $\dot{w}^{-1} x \in$ $\breve{B}_{1}$, and we are done.

We now prove the assertion for $r \geq 2$. By the above, we see that there exists a unique $w \in W_{\mathbf{x}}(T)$ such that $x \in \dot{w} \breve{B}_{r} \breve{G}_{r}^{1}$. We proceed by induction; to this end, it suffices to prove that if $x \in \dot{w} \breve{B}_{r} \breve{G}_{r}^{r-1}$, then $x \in \dot{w} \breve{B}_{r}$.

Since $\mathbb{G}_{r}^{r-1}$ is normal in $\mathbb{G}_{r}$, we may write $x=\dot{w} h b$ for some $h \in \breve{G}_{r}^{r-1}$ and $b \in \breve{B}_{r}$. By [MP96, Theorem 4.2], $\breve{G}_{r}^{r-1}$ has an Iwahori decomposition, so we may write $h=h_{-} h_{+}$with $h_{-} \in \breve{U}_{r}^{-, r-1}$ and $h_{+} \in \breve{B}_{r}^{r-1}$. Replacing $b$ by $h_{+} b$ and $h$ by $h_{-}$, we now have $h \in \breve{U}_{r}^{-, r-1}$. Since $x^{-1} s x \in \breve{B}_{r}$ by assumption, we have $h^{-1} \operatorname{Ad}\left(w^{-1}\right)(s) h \in \breve{B}_{r}$. Writing $t$ for the very regular element $\operatorname{Ad}\left(w^{-1}\right)(s) \in \breve{T}_{r}$, we deduce $h^{-1}\left(t h t^{-1}\right) t \in \breve{B}_{r}$, and thus $h^{-1}\left(t h t^{-1}\right) \in \breve{B}_{r}$. Since $h \in \breve{U}_{r}^{-, r-1}$ by construction, $h^{-1}\left(t h t^{-1}\right) \in \breve{B}_{r}$ only if $h=t h t^{-1}$, which holds only when $h=1$ by Lemma 5.5

Lemma 5.5. Let $r \geq 2$ and let $t \in \breve{T}_{r} \subset \breve{G}_{r}$ be unramified very regular. If $t h t^{-1}=h$ for some $h \in \breve{U}_{r}$, then $h=1$. 
Proof. Fixing an order on the roots $\Phi^{+}=\Phi(T, U)$, we may write $h$ uniquely as $\prod_{\alpha \in \Phi^{+}} \psi_{\alpha}\left(h_{\alpha}\right)$, where $\psi_{\alpha}$ is an isomorphism of $\mathbb{U}_{\alpha, r}$ with a framing object coming from the Chevalley system. Then

$$
\prod_{\alpha \in \Phi^{+}} \psi_{\alpha}\left(h_{\alpha}\right)=h=\zeta^{-1} h \zeta=\prod_{\alpha \in \Phi^{+}} \psi_{\alpha}\left(\alpha\left(\zeta^{-1}\right) h_{\alpha}\right)
$$

and hence (by uniqueness of the presentation as a product) $h_{\alpha}=\alpha\left(\zeta^{-1}\right) h_{\alpha}$. We have naturally $h_{\alpha} \in \mathfrak{p}^{r_{1, \alpha}} / \mathfrak{p}^{r_{2, \alpha}}$ for appropriate $r_{1, \alpha} \leq r_{2, \alpha} \in \mathbb{Z}$. As $\zeta^{-1}$ is very regular, $\alpha\left(\zeta^{-1}\right) \not \equiv 1 \bmod \mathfrak{p}$, and hence the above equality forces $h_{\alpha}=0$ for all $\alpha$. Thus $h=1$.

By Proposition 5.4 ,

$$
S_{T, U}^{(g, \mathbb{T})}:=\left\{x \in \breve{G}_{r}: x^{-1} \sigma(x) \in \breve{U}_{r} \text { and } g x \in x \breve{T}_{r}^{\sigma}\right\}=\bigsqcup_{w \in W_{\mathbf{x}}\left(T, Z^{0}(g)\right)} S_{T, U}^{(g, \mathbb{T})}(w),
$$

where

$$
S_{T, U}^{(g, \mathbb{T})}(w):=\left\{x \in \ddot{w} \breve{B}_{r}: x^{-1} \sigma(x) \in \breve{U}_{r} \text { and } g x \in x \breve{T}_{r}^{\sigma}\right\},
$$

for some (any) lift $\ddot{w} \in \mathbb{G}_{r}$ of $w$. For any $k$-rational $\breve{k}$-split maximal torus $T^{\prime} \subset G$ whose apartment contains $\mathbf{x}$, the preimage of any $w \in W_{\mathbf{x}}\left(T, T^{\prime}\right)^{\sigma}$ in $\mathbb{G}_{r}$ is an $\mathbb{F}_{q^{-}}$ rational $\mathbb{T}_{r}$-torsor, so by Lang's theorem, it contains a $\mathbb{F}_{q}$-rational point $\dot{w}$. For any $w \in W\left(T, T^{\prime}\right)^{\sigma}$ we fix such a $\dot{w}$.

Proposition 5.6. Let $g \in \breve{G}_{r}^{\sigma}$ be an unramified very regular element. Then

$$
S_{T, U}^{(g, \mathbb{T})}(w)= \begin{cases}\dot{w} \breve{T}_{r}^{\sigma} & \text { if } w \in W_{\mathbf{x}}\left(T, Z^{0}(g)\right)^{\sigma} \\ \varnothing & \text { otherwise. }\end{cases}
$$

Proof. Let $w \in W_{\mathbf{x}}\left(T, Z^{0}(g)\right)$ and let $\ddot{w}$ be any lift of $w$ to $\breve{G}_{r}$. Assume that $S_{T, U}^{(g, \mathbb{T})}(w) \neq \varnothing$ and let $x \in S_{T, U}^{(g, \mathbb{T})}(w)$. Then $\ddot{w}^{-1} x \in \breve{B}_{r}$ and we may write $x=\ddot{w} t v$ with $t \in \breve{T}_{r}$ and $v \in \breve{U}_{r}$. We have $x^{-1} g x=v^{-1} t^{-1} \ddot{w}^{-1} g \ddot{w} t v=v^{-1} s v s^{-1} s \in T_{r}^{\sigma}$, where $s:=\ddot{w}^{-1} g \ddot{w} \in T_{r}^{\sigma}$ is unramified very regular. Then $v^{-1} s v s^{-1} \in T_{r}^{\sigma}$, hence necessarily $v=s v s^{-1}$, which forces $v=1$ by Lemma 5.5 .

We now have $x=\ddot{w} t \in \ddot{w} \breve{T}_{r}$. By construction, $t^{-1} \ddot{w}^{-1} \sigma(\ddot{w}) \sigma(t) \in \breve{U}_{r}$. Since the left-hand side is semisimple, we have $\ddot{w} t=\sigma(\ddot{w} t)$, thus forcing $w \in W_{\mathbf{x}}\left(T, Z^{0}(g)\right)^{\sigma}$ and $S_{T, U}^{(g, \mathbb{T})}(w)=\dot{w} \breve{T}_{r}^{\sigma}$.

Proof of Theorem 1.2. For any $\breve{k}$-split maximal torus $T^{\prime} \subset G$, we have a short exact sequence

$$
1 \rightarrow\left(\breve{T}_{r}^{\prime 1}\right)^{\sigma} \rightarrow \breve{T}_{r}^{\prime \sigma} \rightarrow \breve{T}_{1}^{\prime \sigma} \rightarrow 1
$$

of finite abelian groups with $\left(\breve{T}_{r}^{\prime 1}\right)^{\sigma}$ of $p$-power order and $\breve{T}_{1}^{\prime \sigma}$ of order prime to $p$. (The surjectivity on the right holds as $\breve{T}_{1}^{\prime \sigma} \rightarrow H^{1}\left(\operatorname{Gal}\left(\overline{\mathbb{F}}_{q} / \mathbb{F}_{q}\right), \breve{T}_{r}^{\prime 1}\right)$ must be the zero morphism, as the latter is a $p$-group). This sequence is split.

Applying the above to $T^{\prime}=Z^{0}(g)$, we may write $g=s t_{1}$ where $t_{1} \in\left(\breve{T}_{r}^{\prime 1}\right)^{\sigma}$ has $p$-power order and $s$ is in the image of the splitting and hence of order prime to $p$. It is easy to see that $t_{1}$ and $s$ are both powers of $g$. Note that $s$ is still very regular and $Z^{0}(s)=Z^{0}(g)$. Analogously, applying the above to $T^{\prime}=T$, for any $\tau \in \breve{T}_{r}^{\sigma}$, we may write $\tau=\zeta \tau_{1}$ with $\tau_{1} \in\left(\breve{T}_{r}^{1}\right)^{\sigma}$, and $\zeta$ in the image of the splitting. Thus $(g, \tau) \in \breve{G}_{r}^{\sigma} \times \breve{T}_{r}^{\sigma}$ has the decomposition $(g, \tau)=(s, \zeta) \cdot\left(t_{1}, \tau_{1}\right)$, where $(s, \zeta)$ and $\left(t_{1}, \tau_{1}\right)$ are both powers of $(g, \tau)$ such that $(s, \zeta)$ has prime-to- $p$ order and $\left(t_{1}, \tau_{1}\right)$ has 
$p$-power order. Averaging over $\breve{T}_{r}^{\sigma}$ and applying the Deligne-Lusztig trace formula [DL76, Theorem 3.2] (which we may do by Lemma 3.1), we deduce

$$
\begin{aligned}
\operatorname{Tr}\left(g, R_{T, U, r}^{\theta}\right) & =\frac{1}{\# \breve{T}_{r}^{\sigma}} \sum_{\tau \in \breve{T}_{r}^{\sigma}} \theta(\tau)^{-1} \operatorname{Tr}\left((g, \tau)^{*} ; \sum_{i}(-1)^{i} H_{c}^{i}\left(S_{T, U}, \overline{\mathbb{Q}}_{\ell}\right)\right) \\
& =\frac{1}{\# \breve{T}_{r}^{\sigma}} \sum_{\tau \in \breve{T}_{r}^{\sigma}} \theta(\tau)^{-1} \operatorname{Tr}\left(\left(t_{1}, \tau_{1}\right)^{*} ; \sum_{i}(-1)^{i} H_{c}^{i}\left(S_{T, U}^{(s, \zeta)}, \overline{\mathbb{Q}}_{\ell}\right)\right)
\end{aligned}
$$

where $S_{T, U}^{(s, \zeta)}:=\left\{x \in \mathbb{G}_{r}: x^{-1} \sigma(x) \in \mathbb{U}_{r}, s x \zeta=x\right\}$ is the set of fixed points of $S_{T, U}$ under $(s, \zeta)$.

We obviously have $S_{T, U}^{(s, \zeta)} \subseteq S_{T, U}^{(g, \mathbb{T})}$, and it now follows easily from Proposition 5.6 that

$$
S_{T, U}^{(s, \zeta)}= \begin{cases}\dot{w} \mathbb{T}_{r}^{\sigma} & \text { if } \zeta=\operatorname{Ad}\left(w^{-1}\right)\left(s^{-1}\right) \text { for some (unique) } w \in W_{\mathbf{x}}\left(T, Z^{0}(g)\right)^{\sigma} \\ \varnothing & \text { otherwise. }\end{cases}
$$

Now $\left(t_{1}, \tau_{1}\right)$ acts on a point $\dot{w} a \in \dot{w} \mathbb{T}_{r}^{\sigma}$ by $\left(t_{1}, \tau_{1}\right): \dot{w} a \mapsto t_{1} \dot{w} a \tau_{1}=\dot{w} \operatorname{Ad}\left(w^{-1}\right)\left(t_{1}\right) a \tau_{1}$, and thus

$$
\begin{aligned}
\operatorname{Tr}\left(\left(t_{1}, \tau_{1}\right)^{*} ; \sum_{i}(-1)^{i} H_{c}^{i}\left(S_{T, U}^{(s, \zeta)}, \overline{\mathbb{Q}}_{\ell}\right)\right) & =\operatorname{Tr}\left(\left(t_{1}, \tau_{1}\right)^{*} ; H_{c}^{0}\left(\dot{w} \mathbb{T}_{r}^{\sigma}\right)\right) \\
& = \begin{cases}\# \breve{T}_{r}^{\sigma} & \text { if } \tau_{1}=\operatorname{Ad}\left(w^{-1}\right)\left(t_{1}^{-1}\right) \\
0 & \text { otherwise }\end{cases}
\end{aligned}
$$

and Theorem 1.2 now follows from (5.2).

\section{ACKnowledgments}

We would like to thank the anonymous referees for their helpful comments which have improved the exposition of this paper.

\section{REFERENCES}

[BH05] Colin J. Bushnell and Guy Henniart, The essentially tame local Langlands correspondence. II. Totally ramified representations, Compos. Math. 141 (2005), no. 4, 979-1011, DOI 10.1112/S0010437X05001363. MR2148193

[Bor91] Armand Borel, Linear algebraic groups, 2nd ed., Graduate Texts in Mathematics, vol. 126, Springer-Verlag, New York, 1991. MR.1102012

[BS17] Bhargav Bhatt and Peter Scholze, Projectivity of the Witt vector affine Grassmannian, Invent. Math. 209 (2017), no. 2, 329-423, DOI 10.1007/s00222-016-0710-4. MR3674218

[BT72] F. Bruhat and J. Tits, Groupes réductifs sur un corps local (French), Inst. Hautes Études Sci. Publ. Math. 41 (1972), 5-251. MR327923

[BT84] F. Bruhat and J. Tits, Groupes réductifs sur un corps local. II. Schémas en groupes. Existence d'une donnée radicielle valuée (French), Inst. Hautes Études Sci. Publ. Math. 60 (1984), 197-376. MR756316

[BW13] M. Boyarchenko and J. Weinstein, Geometric realization of special cases of local Langlands and Jacquet-Langlands correspondences, Preprint. arXiv:1303.5795 2013.

[BW16] Mitya Boyarchenko and Jared Weinstein, Maximal varieties and the local Langlands correspondence for $G L(n)$, J. Amer. Math. Soc. 29 (2016), no. 1, 177-236, DOI 10.1090/jams826. MR.3402698

[CI20] C. Chan and A. B. Ivanov. Affine Deligne-Lusztig varieties at infinite level, to appear in Math. Ann., 2020+. arXiv:1811.11204. 
[DL76] P. Deligne and G. Lusztig, Representations of reductive groups over finite fields, Ann. of Math. (2) 103 (1976), no. 1, 103-161, DOI 10.2307/1971021. MR393266

[Gre61] Marvin J. Greenberg, Schemata over local rings, Ann. of Math. (2) 73 (1961), 624-648, DOI 10.2307/1970321. MR 126449

[Gre63] Marvin J. Greenberg, Schemata over local rings. II, Ann. of Math. (2) 78 (1963), 256-266, DOI 10.2307/1970342. MR 156855

[Hen92] Guy Henniart, Correspondance de Langlands-Kazhdan explicite dans le cas non ramifié (French, with French summary), Math. Nachr. 158 (1992), 7-26, DOI 10.1002/mana.19921580102. MR.1235293

[HR08] T. Haines and M. Rapoport, On parahoric subgroups, Appendix to PR08], 2008.

[Lus79] G. Lusztig, Some remarks on the supercuspidal representations of p-adic semisimple groups, Automorphic forms, representations and $L$-functions (Proc. Sympos. Pure Math., Oregon State Univ., Corvallis, Ore., 1977), Proc. Sympos. Pure Math., XXXIII, Amer. Math. Soc., Providence, R.I., 1979, pp. 171-175. MR.546595

[Lus04] G. Lusztig, Representations of reductive groups over finite rings, Represent. Theory 8 (2004), 1-14, DOI 10.1090/S1088-4165-04-00232-8. MR2048585

[MP94] Allen Moy and Gopal Prasad, Unrefined minimal K-types for p-adic groups, Invent. Math. 116 (1994), no. 1-3, 393-408, DOI 10.1007/BF01231566. MR1253198

[MP96] Allen Moy and Gopal Prasad, Jacquet functors and unrefined minimal K-types, Comment. Math. Helv. 71 (1996), no. 1, 98-121, DOI 10.1007/BF02566411. MR1371680

[PR08] G. Pappas and M. Rapoport, Twisted loop groups and their affine flag varieties, Adv. Math. 219 (2008), no. 1, 118-198, DOI 10.1016/j.aim.2008.04.006. With an appendix by T. Haines and Rapoport. MR2435422

[Sta09] Alexander Stasinski, Unramified representations of reductive groups over finite rings, Represent. Theory 13 (2009), 636-656, DOI 10.1090/S1088-4165-09-00350-1. MR2558788

[Sta12] Alexander Stasinski, Reductive group schemes, the Greenberg functor, and associated algebraic groups, J. Pure Appl. Algebra 216 (2012), no. 5, 1092-1101, DOI 10.1016/j.jpaa.2011.10.027. MR 2875329

[Tit79] J. Tits, Reductive groups over local fields, Automorphic forms, representations and $L$ functions (Proc. Sympos. Pure Math., Oregon State Univ., Corvallis, Ore., 1977), Proc. Sympos. Pure Math., XXXIII, Amer. Math. Soc., Providence, R.I., 1979, pp. 29-69. MR.546588

[Yu01] Jiu-Kang Yu, Construction of tame supercuspidal representations, J. Amer. Math. Soc. 14 (2001), no. 3, 579-622, DOI 10.1090/S0894-0347-01-00363-0. MR1824988

[Yu15] Jiu-Kang Yu, Smooth models associated to concave functions in Bruhat-Tits theory (English, with English and French summaries), Autour des schémas en groupes. Vol. III, Panor. Synthèses, vol. 47, Soc. Math. France, Paris, 2015, pp. 227-258. MR 3525846

[Zhu17] Xinwen Zhu, Affine Grassmannians and the geometric Satake in mixed characteristic, Ann. of Math. (2) 185 (2017), no. 2, 403-492, DOI 10.4007/annals.2017.185.2.2. MR.3612002

Department of Mathematics, Princeton University, Fine Hall, Washington Road, Princeton, NeW Jersey 08544-1000

Email address: charchan@mit.edu

Mathematisches Institut, Universität Bonn, Endenicher Allee 60, 53115 Bonn, GerMANY

Email address: ivanov@math.uni-bonn.de 